\title{
Proposition de classement des sources végétales d'acides gras en fonction de leur profil nutritionnel
}

Virginie DUBOIS 1,2

Sylvie BRETON ${ }^{2}$

Michel LINDER ${ }^{1}$

Jacques FANNI ${ }^{1}$

Michel PARMENTIER ${ }^{1}$

${ }^{1}$ Nancy-Université, INPL-ENSAIA,

Laboratoire de science et génie alimentaires

(LSGA),

Nancy-Université,

2 avenue de la Forêt de Haye

BP 172

F-54505, Vandœuvre-les-Nancy cedex, France

$<$ acques.Fanni@ensaia.inpl-nancy.fr>

2 St Hubert, Rungis, France

\begin{abstract}
The role of isolated fatty acids on human health has long been studied by the nutritionists. The consequence of this research for the food industry was a need for a better control of the lipidic profile of manufactured food products. The purpose of the present paper was to give the nutritional profile of 53 vegetable oils in relation with their fatty acid composition and their related nutritional potential. In a first section, the role of isolated fatty acids on blood lipids (LDL-Cholesterol, HDL-Cholesterol and triacylglycerols) has been reviewed. In a second section, the vegetable oils are gathered in relation with their similar fatty acids composition, with the aim of classifying their properties in accordance with their role on blood lipids. From a nutritional point of view, besides the major $\alpha$-linolenic acid source that is rapeseed oil, the classification makes some interesting sources to stand out. They contain either mainly $\alpha$-linolenic acid (camelin, linseed and perilla) or both essential fatty acids as $\alpha$-linolenic and linoleic acids (purslane, chia, salicorna and sea buckthorn seeds). Moreover, this collection is an interesting tool for new designed food products with a better lipid composition.
\end{abstract}

Key words: vegetable sources, blood lipids, fatty acids

\section{Introduction}

L'évolution de la politique nutritionnelle des pouvoirs publics au sujet de l'apport lipidique français a permis de mettre en avant la notion de «qualité de lipides» au lieu de la seule «quantité de lipides ». En effet, l'impact nutritionnel des lipides alimentaires varie fortement en fonction de leur structure, observation qui a été confirmée par les nombreuses études cliniques réalisées durant les dernières décennies [1-9].

Avec ce nouvel intérêt pour les acides gras individualisés, les industriels de l'agroalimentaire sont à la recherche d'huiles particulières pour optimiser le profil lipidique de leurs produits. Un nouveau marché émerge, avec des fournisseurs qui proposent de plus en plus d'huiles spécifiques, chacune ayant une composition en acides gras particulière.

Les recherches bibliographiques effectuées sur les huiles végétales ont permis de créer une base de données contenant les compositions en acides gras de 53 huiles végétales, regroupées en classes de même profil général en acides gras.

Les huiles ont été sélectionnées par une recherche sur Science Direct, en utilisant des mots-clés tels que " profil en acides gras ", " huiles végétales", ou encore " composition en lipides des huiles». Seules les données complètes issues de publications scientifiques ont été incluses dans l'analyse, certains auteurs n'ayant en effet étudié qu'un type d'acides gras, comme les acides gras polyinsaturés (AGPI). C'est pourquoi des huiles mineures, mais disponibles dans des circuits de distribution "diététiques", peuvent ne pas être incluses, tandis que d'autres, non disponibles, le sont.

Les huiles les plus largement utilisées actuellement dans le monde sont les huiles de soja, palme, colza et tournesol [10]. Parmi elles, les huiles de soja et de colza apportent des quantités intéressantes d'acide $\alpha$-linolénique (ALA) - respectivement 7,8 \% et 9,9\% - l'acide gras considéré comme étant le plus déficient dans l'alimentation occidentale par les agences sanitaires européennes [11]. Pour permettre l'identification de toutes les huiles potentiellement intéressantes dans la classification qui suit, un sous-groupe a été créé dans chaque groupe contenant des huiles avec plus de 7,8 \% d'ALA. La teneur en ALA de l'huile de soja est donc utilisée ici comme un seuil au-dessus duquel une huile devient particulièrement intéressante du point de vue nutritionnel.

Depuis plusieurs décennies, les différentes classes de lipides sanguins sont considérées comme étant des facteurs de risque indépendants des accidents cardiovasculaires, qui représentent à I'heure actuelle la première cause de décès en France. Les paramètres concernés sont le LDLcholestérol (« mauvais cholestérol »), le HDL-cholestérol (« bon cholestérol») et les triglycérides plasmatiques (TAGp) [12-19]. La structure des acides gras ingérés influence le métabolisme des lipoprotéines, et donc la concentration en lipides sanguins.

En dehors de cet impact des différentes classes d'acides gras sur la lipémie sanguine, la proportion respective des familles $n$ - 3 et $n-6$ a également une influence sur l'organisme. En effet, les métabolites issus de ces deux familles ont des activités antagonistes, ceux issus des AG n-6 étant

\section{FONDAMENTAI}

Article reçu le 4 décembre 2007 accepté le 29 décembre 2007 
pro-inflammatoires, prothrombotiques et hypertenseurs, tandis que ceux issus des AG n-3 sont anti-inflammatoires, antithrombotiques et hypotenseurs. Les enzymes responsables de la synthèse de ces métabolites étant les mêmes dans le cas des deux familles d'acides gras, un excès de l'un des deux groupes conduit à un déséquilibre entre ces deux séries de métabolites, et donc à une modification entre autres du «statut inflammatoire » de l'organisme [20-22].

Ce sont donc ces deux points, action sur la lipémie sanguine et ratio $n-6 / n-3$, qui seront utilisés comme des clés pour interpréter chaque groupe d'huiles.

\section{Impact des différentes classes de lipides sur les lipides sanguins}

\section{Acides gras saturés (AGS)}

Deux hypothèses ont été avancées pour expliquer l'effet hypercholestérolémiant des ACS :

i) Ils pourraient être responsables de la diminution de l'expression du récepteur hépatique des particules $L D L$, empêchant une récupération efficace de ces lipoprotéines par le foie, ce qui aurait pour conséquence d'augmenter la concentration plasmatique en LDL-cholestérol [23].

ii) Leur incorporation dans les phospholipides membranaires pourrait rigidifier la membrane hépatocytaire du fait de leur point de fusion élevé. Celle-ci perdrait ainsi de sa fluidité, et le fonctionnement des récepteurs aux LDL pourrait en être perturbé [23].

\section{Acides laurique et myristique}

Les acides laurique et myristique augmentent le LDL-cholestérol $[4,7,8$, 24]. L'acide myristique semble plus délétère que le laurique, mais la position d'estérification sur le squelette glycérique semble avoir une importance prépondérante dans le cas de l'impact de cet acide gras. En effet, l'acide myristique serait moins hypercholestérolémiant en position $s n-2$, comme c'est le cas dans la matière grasse laitière [4, 25], qu'en sn-1 ou 3 [26]. De plus, dans cette configuration il semble que l'acide myristique stimule la conversion d'ALA en EPA et DHA, ses dérivés à longue chaîne (acides eicosapentaénoïque et docosahexénoïque, respectivement) [26].

\section{Abréviations}

AFSSA : Agence française de sécurité sanitaire des aliments ALA : acide $\alpha$-linolénique

CSHPF : Conseil supérieur d'hygiène publique de France

DGCCRF : Direction générale du commerce, de la concurrence et de la répression des fraudes

Dihomo-GLA : acide dihomo- $\gamma$-linolénique

EFSA : European Food Safety Agency

GLA : acide $\gamma$-linolénique

HDL-cholestérol : cholestérol des lipoprotéines de forte densité (high density lipoprotein)

LA : acide linoléique

LDL-cholestérol : cholestérol des lipoprotéines de faible densité (low density lipoprotein)

LRA : acide laurique

$M A$ : acide myristique

$\mathrm{OA}$ : acide oléique

PA : acide palmitique

$S A$ : acide stéarique

TAGp : triglycérides plasmatiques

\section{Acide palmitique}

L'acide palmitique est particulier. En effet, si la majorité des études a montré un effet hypercholestérolémiant de cet acide gras $[1,4,7,8]$, d'autres ont démontré une relative neutralité $[24,27]$. Les auteurs de ces deux dernières études émettent l'hypothèse d'un seuil. Ils postulent qu'au-delà de 400 mg de cholestérol alimentaire ingéré par jour, l'acide palmitique augmenterait le taux de LDL-cholestérol (de manière peutêtre supérieure à l'acide myristique), tandis qu'il serait plutôt neutre en deçà $[24,27]$. II semble que des études supplémentaires soient nécessaires pour expliquer la disparité entre ces résultats.

Concernant la position de l'acide palmitique sur le squelette glycérique des triacylglycérols, il augmenterait plus le LDL-cholestérol sanguin en étant placé en position interne [8], ce qui est le cas dans la matière grasse laitière $[4,25]$ ou le lait maternel [28].

\section{Acide stéarique}

L'acide stéarique semble n'avoir aucun effet délétère sur les lipides sanguins [1, 2, 5, 7, 8, 23]. En fait, il a été prouvé qu'un régime riche, en acide stéarique n'augmente pas les lipides plasmatiques comparé à un régime riche, soit en acide oléique, soit en acide linoléique [5].

Deux hypothèses ont été avancées pour expliquer ce phénomène :

i) Le point de fusion de l'acide stéarique libre est bien supérieur à la température du corps humain $\left(70^{\circ} \mathrm{C},[9]\right)$, ce qui fait qu'il est solide à $37^{\circ} \mathrm{C}$, et qu'il est probablement susceptible d'être éliminé dans les fèces au lieu d'être absorbé par l'épithélium intestinal ;

Remarque : Pendant le processus de digestion, la lipase libère spécifiquement les acides gras placés aux positions extérieures des triacylglycérols (position préférentielle des AGS dans les huiles végétales), en laissant l'acide gras interne en place. L'acide stéarique libéré par la lipase se retrouve donc dans la lumière intestinale, à l'état solide du fait de son point de fusion élevé [29].

ii) L'acide stéarique peut être rapidement désaturé en acide oléique, et donc son éventuel effet propre serait masqué [1].

\section{Acides gras monoinsaturés (AGMI)}

L'acide oléique $(\mathrm{OA})$ est l'acide gras majoritaire de nombreuses huiles végétales, en particulier des huiles d'olive et de colza, et est I'AGMI alimentaire principal. II montre selon les études un effet neutre ou légèrement positif vis-à-vis du cholestérol total et du LDL-cholestérol $[1-3,5,7,23,30,31]$.

L'huile d'olive a longtemps été considérée comme « saine » du fait de la proportion d'OA qu'elle contient, mais il semble à présent que d'autres composés de l'huile pourraient être actifs. L'action positive des AGMI sur les lipides sanguins a en effet été démontrée par des études dans lesquelles le régime témoin était composé d'ACS. Ce choix a introduit un biais dans les résultats du fait de l'action délétère de ces acides gras sur le LDL-cholestérol [1, 7, 23]. Quand l'effet des AGMI a été étudié contre un régime riche en glucides, il a été prouvé que ces acides gras sont neutres vis-à-vis des lipides sanguins [2, 3].

À part I'action sur le LDL-cholestérol, il semble que l'acide oléique soit neutre vis-à-vis des TAGp et du HDL-cholestérol [2 et 3, 7, respectivement].

\section{Acides gras polyinsaturés (AGPI)}

\section{Acide linoléique}

Parmi les AGPI, I'acide linoléique (LA), précurseur de la famille des AG $n-6$, est le seul qui semble diminuer le taux de LDL-cholestérol $[7,17,27]$. Certains auteurs nuancent ces résultats, en démontrant que le LA est neutre vis-à-vis du LDL-cholestérol $[5,23]$. D'autres avancent I'hypothèse d'une valeur seuil, au-delà de laquelle cet acide gras deviendrait neutre 
$[24,32]$. D'après eux, I'effet positif sur le cholestérol n'est visible que pour un apport compris entre 10 et $17 \%$ des lipides totaux [32]. Quoi qu'il en soit, les acides gras témoins dans la majorité de ces études sont des AGS, et la seule conclusion acceptable dans ce cas est que remplacer des AGS par du LA permet de diminuer la concentration en LDL-cholestérol [33-35].

Certains auteurs proposent une autre hypothèse, selon laquelle l'un des métabolites du LA, vraisemblablement l'acide $\gamma$-linolénique (GLA) ou I'acide dihomo- $\gamma$-linolénique (dihomo-GLA), serait actif à la place du LA [36]. Sachant que la $\Delta 6$-désaturation (transformation du LA en GLA) est l'étape limitante de la conversion du LA [36], il semble évident que si le dihomo-GLA est le composé actif, apporter directement du GLA soit plus efficace qu'apporter du LA. De plus, un excès de LA inhibe sa propre conversion en dérivés à longue chaîne [37]. Ceci permet de renforcer les deux hypothèses présentées dans les différentes études :

i) Le GLA ou le dihomo-GLA est actif au lieu du LA.

ii) Apporter du LA en excès inhibe l'effet positif observé sur le LDLcholestérol.

Le LA semble diminuer le taux de HDL-cholestérol de manière concomitante à la baisse du LDL-cholestérol [34]. Le LA diminue également, mais de manière faible, le taux de TAGp [17].

Enfin, comme décrit précédemment, un excès de LA dans le régime alimentaire provoque un état pro-inflammatoire dans l'organisme [20-22]. C'est pourquoi le ratio $n-6 / n-3$ recommandé - inférieur ou égal à 5 - préconise que la consommation de LA dans nos régimes occidentaux soit diminuée [11].

\section{Acide a-linolénique}

L'ALA semble neutre vis-à-vis du LDL-cholestérol [38-41]. Pourtant, certaines études ont montré un effet positif de ce précurseur sur le LDLcholestérol, mais dans des tests où d'autres acides gras du régime ont été modifiés en même temps, comme par exemple le LA [42], les AGS [43-45], ou même le régime entier [46, 47].

L'action du précurseur de la série $n-3$ sur le HDL-cholestérol et les TAGp n'est pas plus claire. Certaines études n'ont montré aucun effet [38-40, $42,43]$, deux ont trouvé une diminution des TAGp et du HDL-cholestérol $[44,45]$, une étude reporte une diminution du HDL-cholestérol seulement [41], et deux ont montré une diminution des TAGp et une augmentation du HDL-cholestérol [46, 47]. De même que pour les résultats concernant le LDL-cholestérol, la plupart de ces études ne concernaient pas uniquement une modification de l'apport en ALA, mais une modification plus importante du régime.

II paraît évident que des études mieux contrôlées sont nécessaires pour permettre de tirer des conclusions fermes quant à l'effet du précurseur sur les lipides sanguins. L'agence alimentaire britannique (UK Food Standard Agency) est parvenue à la même remarque dans son rapport sur la comparaison de l'impact de l'ALA par rapport à ses dérivés à longue chaîne, EPA et DHA [48].

Malgré ces conclusions sur l'impact de l'ALA sur la lipémie sanguine, il est important que rappeler que l'ALA est un acide gras essentiel, qui est impliqué dans de nombreuses voies métaboliques. Or les apports en ALA, et en AG $n$-3 en général, sont insuffisants dans le régime alimentaire français [11]. La consommation en ALA doit donc être augmentée pour : i) Rééquilibrer la chaîne de conversion élongation-désaturation vers la production des AGPI n-3 terminaux.

ii) Stimuler la production de composés anti-inflammatoires grâce à la conversion de I'ALA en EPA.

iii) Réguler la production des métabolites issus du LA, et ainsi éviter que le déséquilibre du ratio $n-6 / n-3$ ne provoque un statut pro-inflammatoire [20-22].

\section{Classification des huiles}

\section{Groupe acides gras saturés}

\section{Sous-groupe acide caprique}

Ce type de profil n'est pas courant. En effet, seules deux huiles ont été incluses dans ce groupe : deux sous-espèces de Cupheae (Cuphea ilavea et leptopoda [49], figure 1). Elles contiennent majoritairement des AGS à courte chaîne, en particulier de l'acide caprique (10:0), jusqu'à 92 \% pour Cuphea leptopoda. Leur profil est intéressant dans le cas d'un besoin en lipides rapidement convertis en énergie $[7,23]$.

\section{Sous-groupe acides laurique et myristique}

Les huiles de noix de coco (Cocus nucifera [50-53], figure 2) et de palmiste (Elaeis guineensis $[52,54]$ ) ont deux profils semblables, incluant une majorité de AGS, en particulier de l'acide laurique (12:0).

Ces huiles ne sont pas recherchées d'un point de vue nutritionnel, puisque les acides laurique et myristique sont connus comme étant les AGS les plus hypercholestérolémiants [4, 7, 8, 24-26, 55]. Elles sont pourtant utilisées dans l'industrie agroalimentaire pour maîtriser la texture des produits, comme par exemple dans les matières grasses à tartiner.

\section{Sous-groupe acide palmitique}

Le meilleur exemple de ce groupe est l'huile de palme (Elaeis guineensis $[53,56-58]$, figure 3$)$, couramment utilisée dans les formulations alimentaires pour donner de la texture aux produits.

L'huile de noix pili (Canarium ovatum [59]), quoique moins connue, a le même profil en acides gras. Ces huiles sont plus intéressantes que les précédentes, car l'acide palmitique est moins délétère que les acides laurique ou myristique $[1,4,7,8,24,27]$, en particulier quand il est estérifié sur la position externe du glycérol [8], comme c'est le cas dans ces huiles.

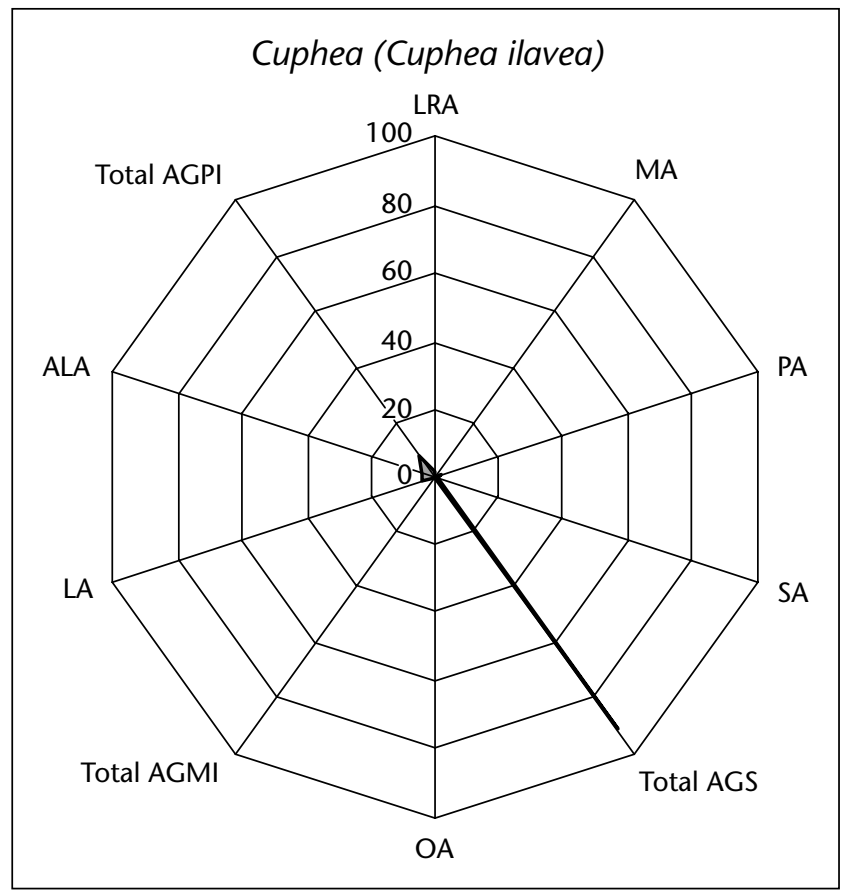

Figure 1. Profil en acides gras de I'huile de Cuphea ilavea. 


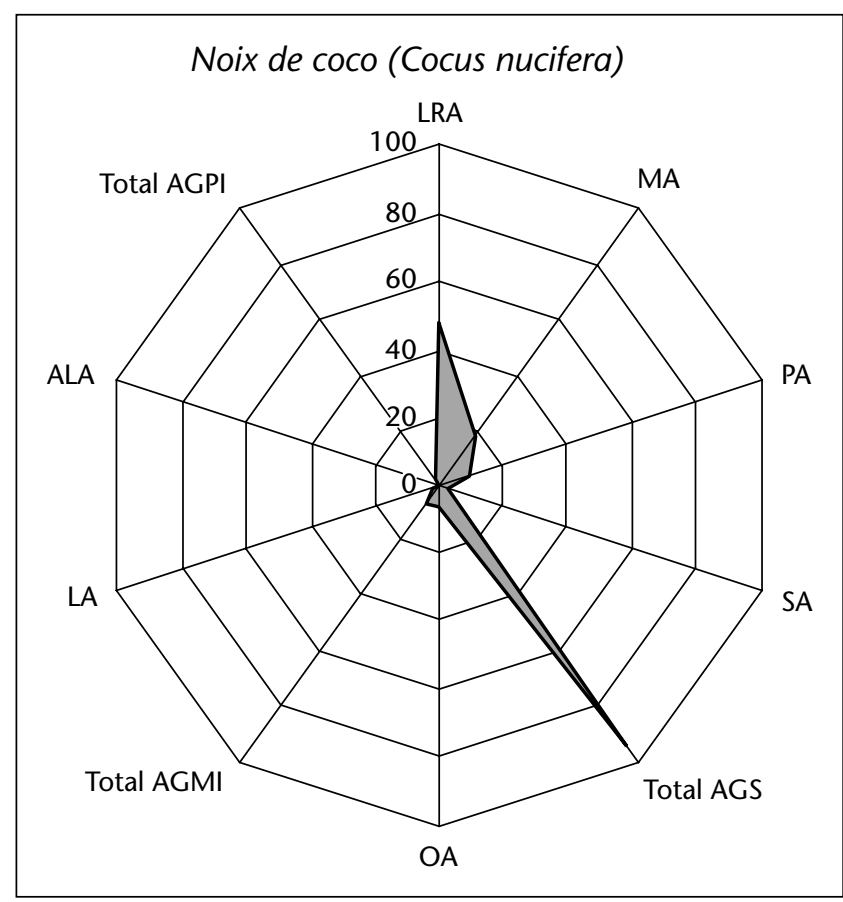

Figure 2. Profil en acides gras de I'huile de noix de coco.

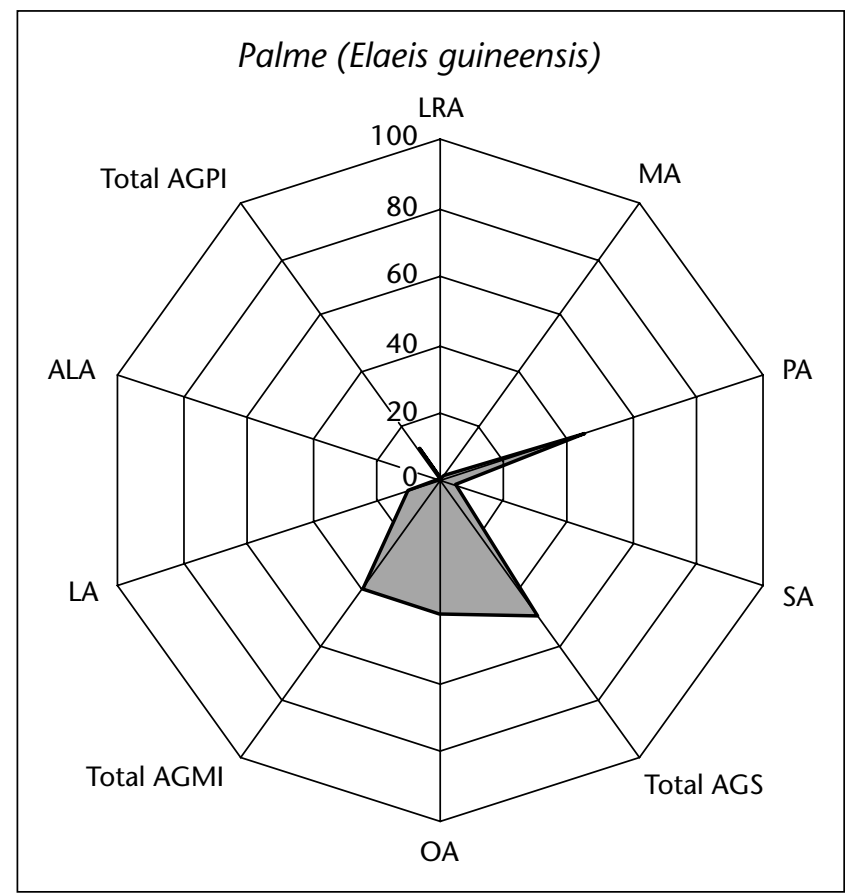

Figure 3. Profil en acides gras de I'huile de palme.

\section{Sous-groupe acide stéarique}

Trois huiles sont incluses dans ce groupe, caractérisées par un profil majoritairement saturé, avec en acide gras principal l'acide stéarique. Ce sont les beurres de cacao (Theobroma cacao [60], figure 4) et de karité (Vitellaria paradoxa - anciennement Butyrospermum parkii - [60]), et I'huile d'amande de mangue (Mangifera indica [61, 62]). Le beurre de cacao est utilisé principalement par l'industrie alimentaire, tandis que le

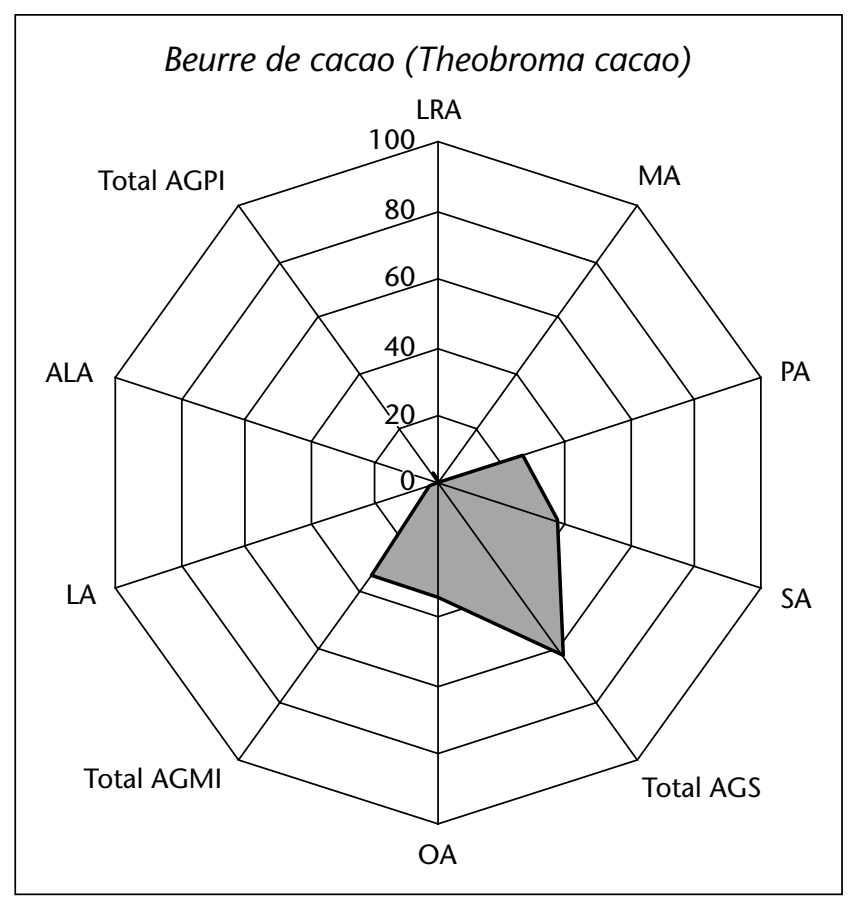

Figure 4. Profil en acides gras du beurre de cacao.

beurre de karité est rencontré dans des produits cosmétiques, mais aussi alimentaires puisque son utilisation en tant qu'alternative au beurre de cacao a été autorisée en France [60].

L'intérêt nutritionnel de ces sources réside dans la neutralité des acides stéarique et oléique (le deuxième composé majoritaire des huiles de ce profil) vis-à-vis des lipides sanguins $[1,2,5,7,8,23]$.

\section{Groupe acides gras mono-insaturés}

\section{Sous-groupe AGMI (> $60 \%$ )}

Le meilleur représentant de ce groupe à profil mono-insaturé est bien évidemment l'huile d'olive (Olea europaea [53, 63-67], figure 5), qui contient presque $80 \%$ d'OA. Les huiles de noisette (Corylus avellana [68, 69]), de noix de macadamia (Macadamia tetraphylla [70]), d'avocat (Persea americana [71]) et de crambe (Crambe abyssinica [72]) ont le même type de profil.

Comme vu dans un chapitre précédent, I'OA est neutre vis-à-vis des lipides sanguins, en particulier du LDL-cholestérol [2, 3, 5, 30, 31]. Utiliser une huile comme celles de ce groupe permet donc de compléter une formulation, à la manière d'un excipient.

Ce groupe est le premier dans lequel on retrouve des huiles ayant la même composition de base, mais complétée par plus de 7,8 \% d'ALA. Dans ce sous-groupe « riche en ALA » sont classées les huiles de colza (Brassica napus [64, 73, 74], figure 5) et de graine de moutarde blanche (Brassica alba [53,69]). Elles contiennent respectivement 9,9\% et $10,3 \%$ d'ALA, et associent donc la neutralité de l'OA à la présence de I'ALA [20-22].

Deux huiles font partie de ce groupe malgré un profil inhabituel. En effet, les huiles de crambe et de graine de moutarde blanche contiennent de I'acide érucique (22:1 n-9) comme AGMI majoritaire, au lieu de I'OA. Cet acide gras, donné chez l'animal à des doses importantes, a été soupçonné d'avoir des effets délétères sur la santé [75]. Cette polémique a engendré la sélection d'une variété de colza sans acide érucique (ni glucosinolate, un autre composé néfaste pour l'alimentation animale utilisatrice des tourteaux) : le colza 0:0, ou canola. 


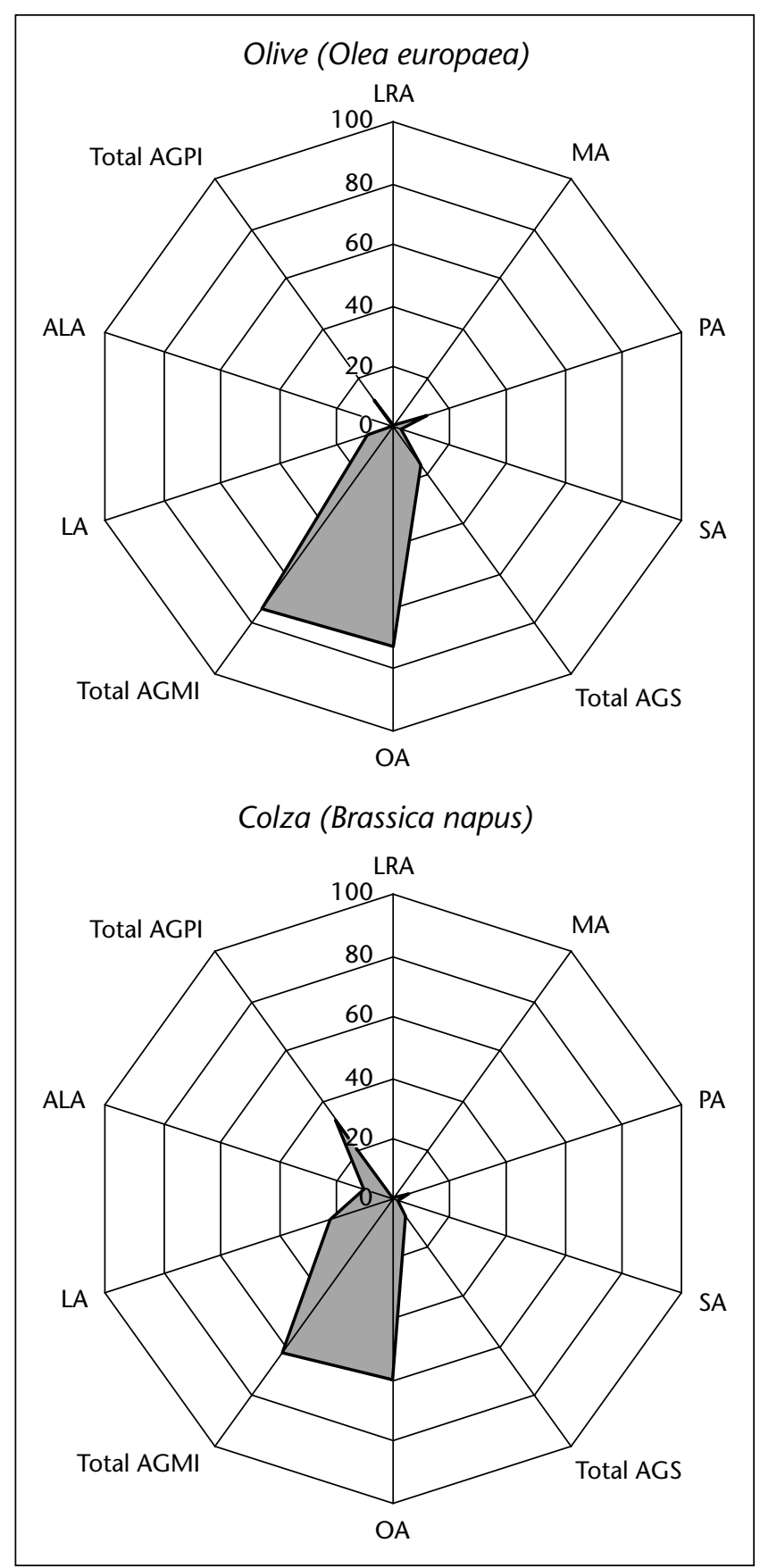

Figure 5. Profil en acides gras des huiles d'olive (haut) et de colza (bas).

\section{Sous-groupe AGMI+AGS+LA}

Ce groupe est caractérisé par un profil en acides gras contenant moins d'AGMI que le précédent, avec du LA en remplacement. II comprend les huiles d'arachide (Arachis hypogea [69, 74, 76-78], figure 6), d'avoine (Avena sativa [79]), de sarrasin (Polygonum fagopyrum [80]), de pistache (Pistacia atlantica [81]) et d'argan (Argania spinosa [82]).

Le sous-groupe « riche en ALA » comprend I'huile de baie d'argousier - 8,8 \% - (Hippophae rhamnoides $[83,84]$ ). Cette dernière montre un profil très intéressant, car elle apporte une proportion non négligeable de précurseur des AG $n-3$ avec moins de $20 \%$ de LA (15,65\%), ce qui lui confère un ratio $n-6 / n-3$ inférieur à 2 .

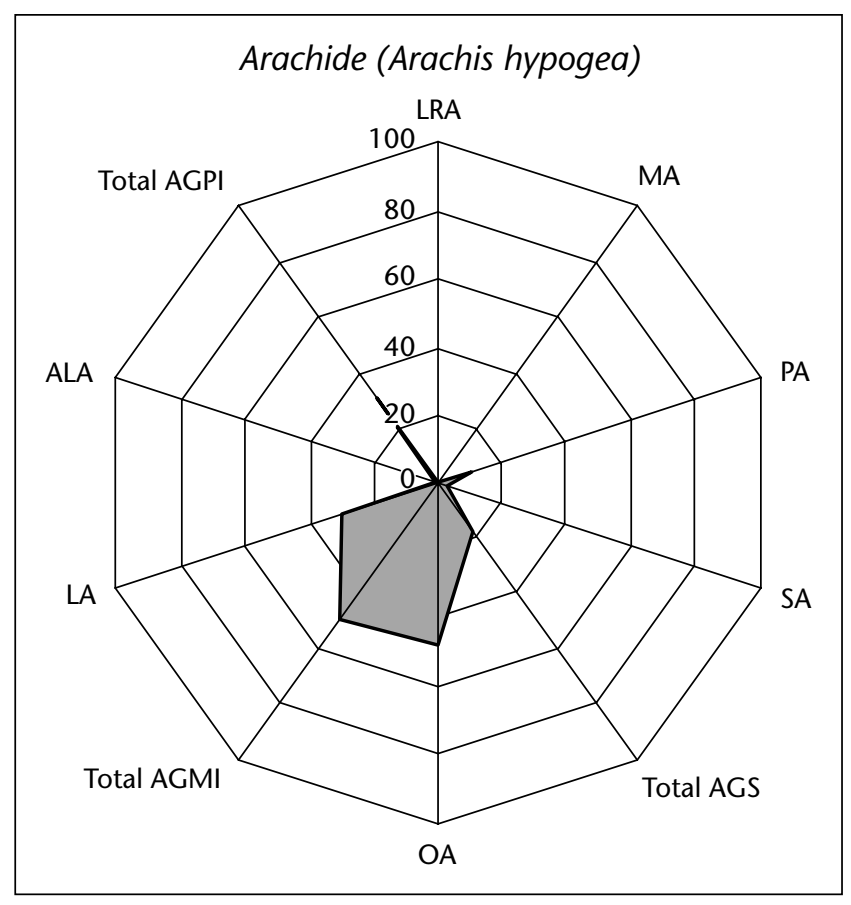

Figure 6. Profil en acides gras de l'huile d'arachide.

\section{Groupe acides gras polyinsaturés}

\section{Sous-groupe $L A(>60 \%)$}

Les huiles de ce groupe comprennent une majorité d'AGPI, dont plus de $60 \%$ de LA. En gardant à l'esprit qu'un excès d'AG $n-6$ provoque un état pro-inflammatoire dans l'organisme [20-22], ces huiles semblent être trop riches en LA pour être intéressantes. Cela rend complexe leur utilisation en formulation, à cause du ratio $n-6 / n-3$, dont la valeur recommandée inférieure à 5 semble alors difficile à maintenir [11]. La seule alternative permettant leur utilisation est de les mélanger avec des huiles de profils différents.

Les produits rassemblés dans ce groupe sont les huiles de pépin de raisin (Vitis vinifera $[53,62,85,86]$, figure 7 ), d'onagre (Oenothera biennis [53, 87-89]), de pépin de melon (Cucumis melo [90]), de carthame (Carthamus tintorius [53, 69, 91-93]) et de salicorne (Salicornia bigelovii [91]).

L'huile de pépin de cassis (Ribes nigrum [86]) contient plus de 7,8\% d'ALA en complément de cette grande quantité de LA $(11,9 \%)$. De plus, cette matière première contient plus de $12 \%$ de GLA [86], qui pourrait être le composé hypocholestérolémiant de la famille $n-6$, selon Horrobin et Huang (1987) [36]. Au contraire, I'huile d'onagre contient également une proportion importante de GLA, mais quasiment pas d'ALA.

Ce niveau de précurseur des AG $n-3$ dans l'huile de pépin de cassis en fait le produit le plus intéressant de ce groupe car son ratio $n-6 / n-3$ est abaissé à 4,4 par cet apport en ALA. II est malgré tout nécessaire de rappeler que la consommation d'AG $n-6$ dans la population française est excédentaire, et que les dérivés pro-inflammatoires générés par ces acides gras imposent certaines précautions quant à l'utilisation d'une telle huile.

\section{Sous-groupe LA+AGS}

Le profil des produits de ce groupe contient majoritairement du LA, additionné d'AGS (principalement palmitique), et, comme dans le cas précédent, ils ne paraissent pas intéressants utilisés seuls $[1,4,7,8,24$, 32]. Sont retrouvées dans ce groupe les huiles de graine de coton (Gossypium hirsutum [53, 69, 74, 80, 94, 95], figure 8), de cumin noir 


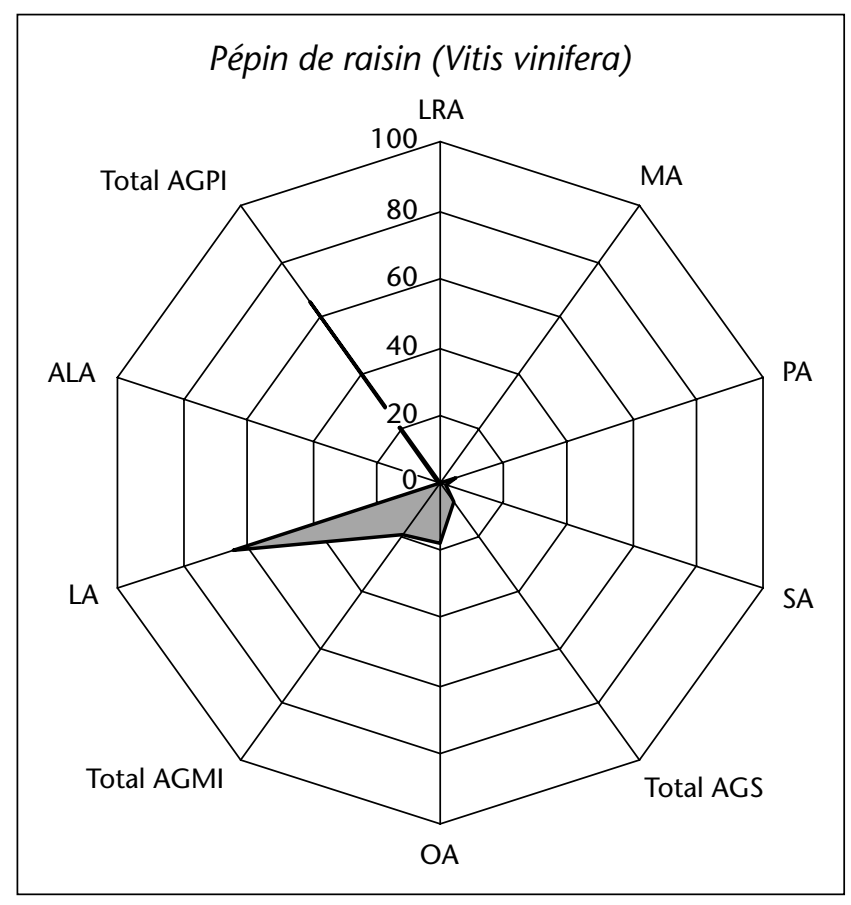

Figure 7. Profil en acides gras de l'huile de pépin de raisin.

(Nigella sativa [96]), de pépin de pastèque (Citrullus sp. [97]) et d'amarante (Amaranthus hypocondriacus [80]).

Les huiles de noix (Juglans regia [98], figure 8) et de lupin (Lupinus mexicanus [99]) montrent un profil comparable, avec en supplément $10 \%$ et $8 \% d^{\prime} A L A$, respectivement.

\section{Sous-groupe LA+AGMI}

L'huile de tournesol (Helianthus annuus [53, 57, 69, 74, 100, 101], figure 9) contient principalement du LA et de I'OA. Les huiles suivantes ont le même profil : maïs (Zea mays [53, 64, 69, 80, 102-104]), germe de blé (Triticum aestivum $[53,105,106])$, sésame (Sesamum indicum $[53,64,69$, 80, 107-111]), cumin (Cuminum cymimum [112]), bourrache (Borago officinalis $[88,89,113])$ et deux sous-espèces d'amarante (Amaranthus caudatus [114] et Amaranthus cruentus [80]).

Parmi ces sources, I'huile de bourrache se distingue par sa teneur en GLA, qui atteint $22 \%$ des acides gras totaux $[88,89,113]$. Comme pour I'huile de pépin de cassis dans le sous-groupe précédent, l'huile de bourrache pourrait avoir un effet hypocholestérolémiant grâce à cette particularité de composition [36].

Quelques huiles ont le même profil que celles citées ci-dessus, mais additionné de plus de 7,8\% d'ALA. Ce sont les huiles de soja (Glycine max $[53,64,69,74,80,115]$, figure 9), de chanvre (Cannabis sativa [53, 112, 116]), de fénugrec (Trigonella foenum-graecum [117]) et de pépin de canneberge (Vaccinium oxycoccos [112]). Ces quatre produits contiennent respectivement $7,8 \%, 19,7 \%, 23,2 \%$ et $22,3 \%$ de précurseur des $n$-3, ce qui leur confère des ratios $n-6 / n-3$ compris entre 1,8 et 6,7 .

$\mathrm{Si}$ une huile riche en LA est nécessaire pour préparer un mélange, ces dernières devront être préférées à celles des sous-groupes LA et LA+AGS, puisqu'elles apportent de I'ALA plutôt que des AGS $[2,3]$.

\section{Sous-groupe ALA+AGMI}

Ces deux derniers groupes sont dédiés aux huiles contenant en majorité de I'ALA, de $28 \%$ à presque $55 \%$ des acides gras totaux. Cet acide gras représente une cible nutritionnelle importante, puisque sa conversion en EPA permet de contrecarrer les effets délétères d'un régime alimentaire

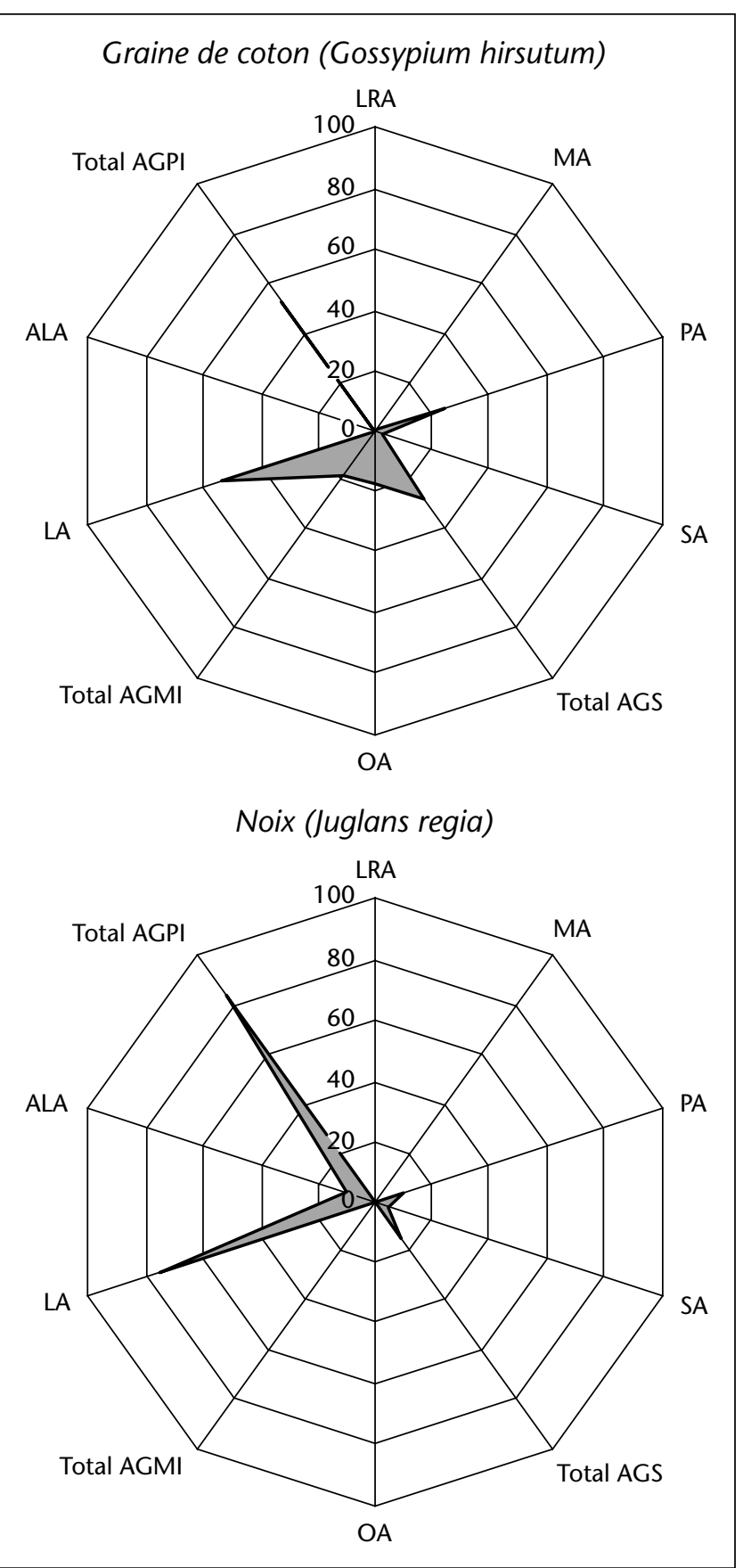

Figure 8. Profil en acides gras des huiles de graine de coton (haut) et de noix (bas).

trop riche en AG $n-6$ [20-22]. L'utilisation de telles sources d'ALA en formulation permettrait de ramener aisément le rapport $n-6 / n-3$ dans des limites compatibles avec les recommandations de I'AFSSA.

Dans ce premier groupe, les huiles contiennent en majorité des AGMI en plus du précurseur des $n-3$. Ce sont les huiles de cameline (Camelina sativa [118]), de lin (Linum usitatissimum [53, 93, 111], figure 10) et de périlla (Perilla frutescens $[64,119])$. Parmi elles, les huiles de lin et de périlla sont les plus riches en ALA, avec des valeurs de $55 \%$ chacune, contre $38,1 \%$ pour la cameline. 


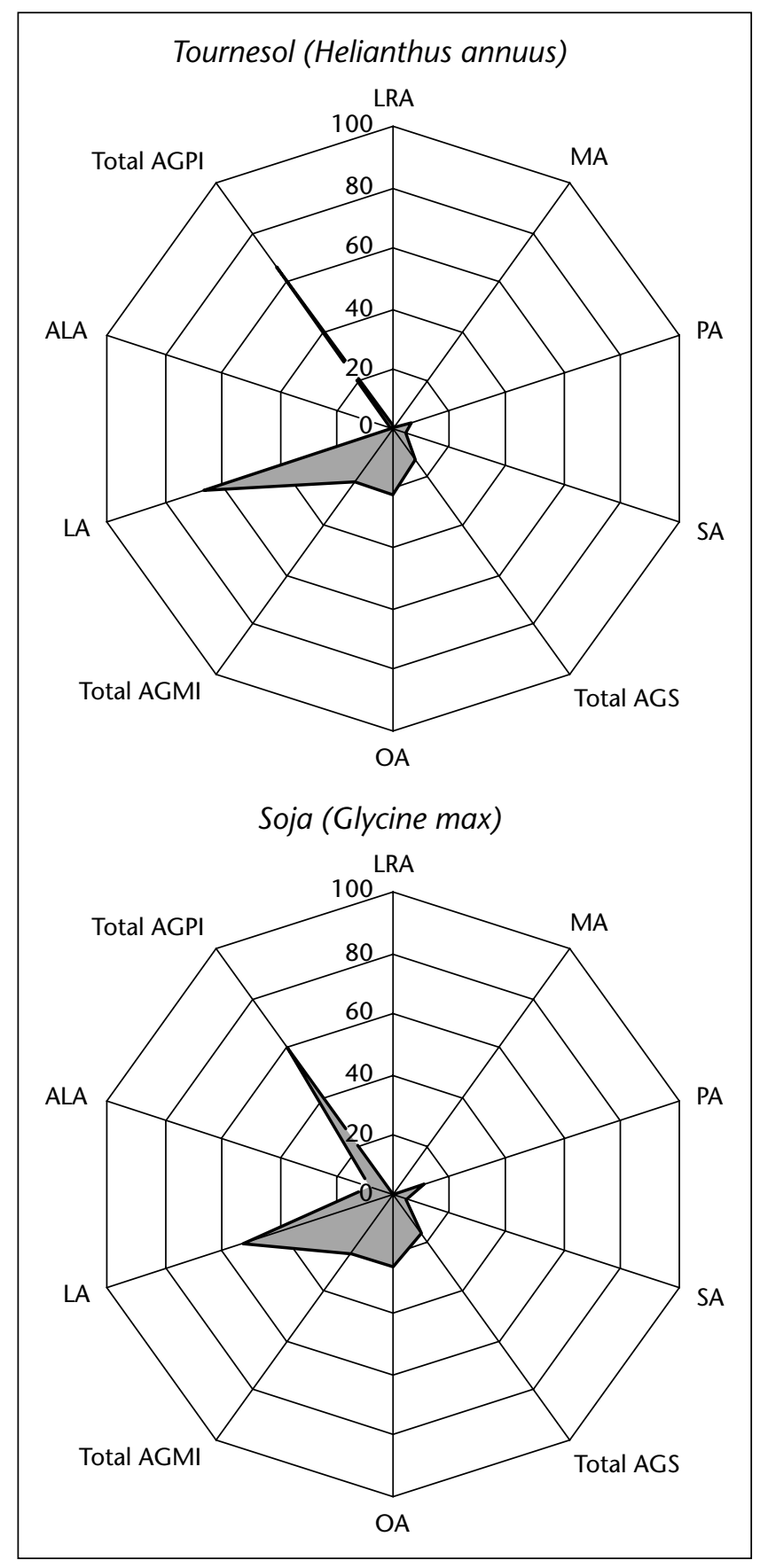

Figure 9. Profil en acides gras des huiles de tournesol (haut) et de soja (bas).

\section{Sous-groupe ALA+LA}

Le dernier groupe de ce classement comprend les huiles de pourpier (Portulaca oleracea $[120,121]$, figure 11), de chia (Salvia hispanica [87, $122,123])$, de graine d'argousier (Hippophae rhamnoides [83, 84]) et de salicorne (Salicornia europaea [120]).

Le profil des produits de ce groupe associe un niveau d'ALA supérieur à $28 \%$ (pourpier : $32,4 \%$, chia : $61,3 \%$, graine d'argousier : $28,8 \%$, salicorne : $28 \%$ ), avec $20 \%$ ou plus de LA. Avec des ratios $n-6 / n-3$ inférieurs à 2, ces huiles sont très intéressantes car elles permettent d'apporter les deux acides gras essentiels sous un rapport avantageux dans une optique de rééquilibrage.

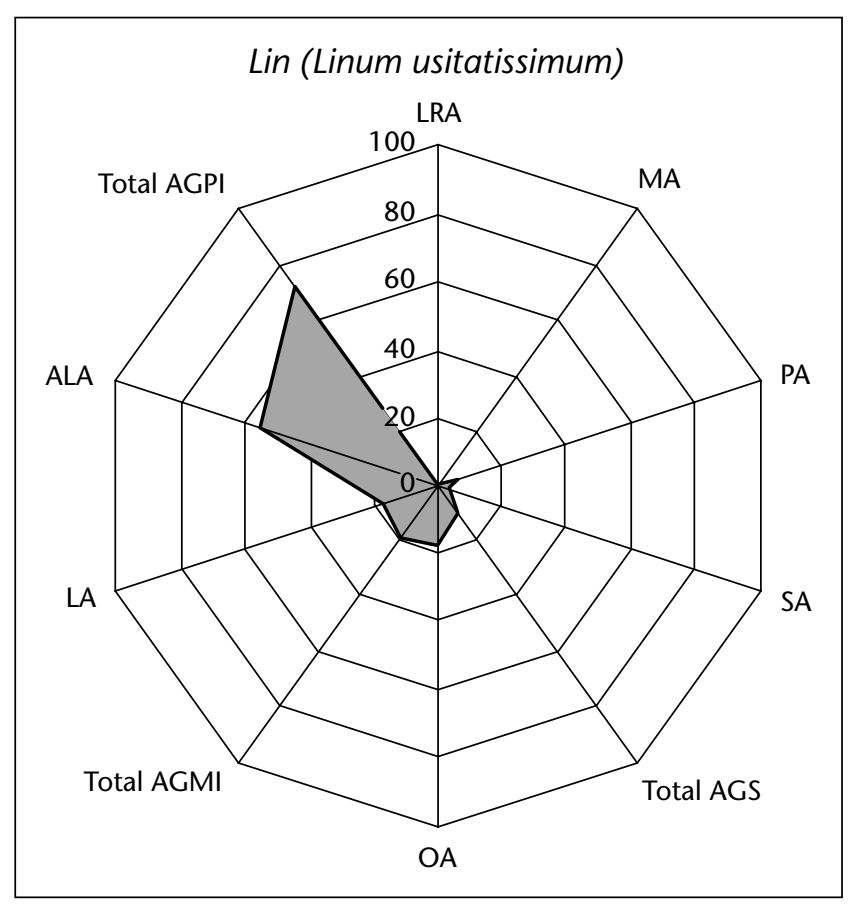

Figure 10. Profil en acides gras de I'huile de lin.

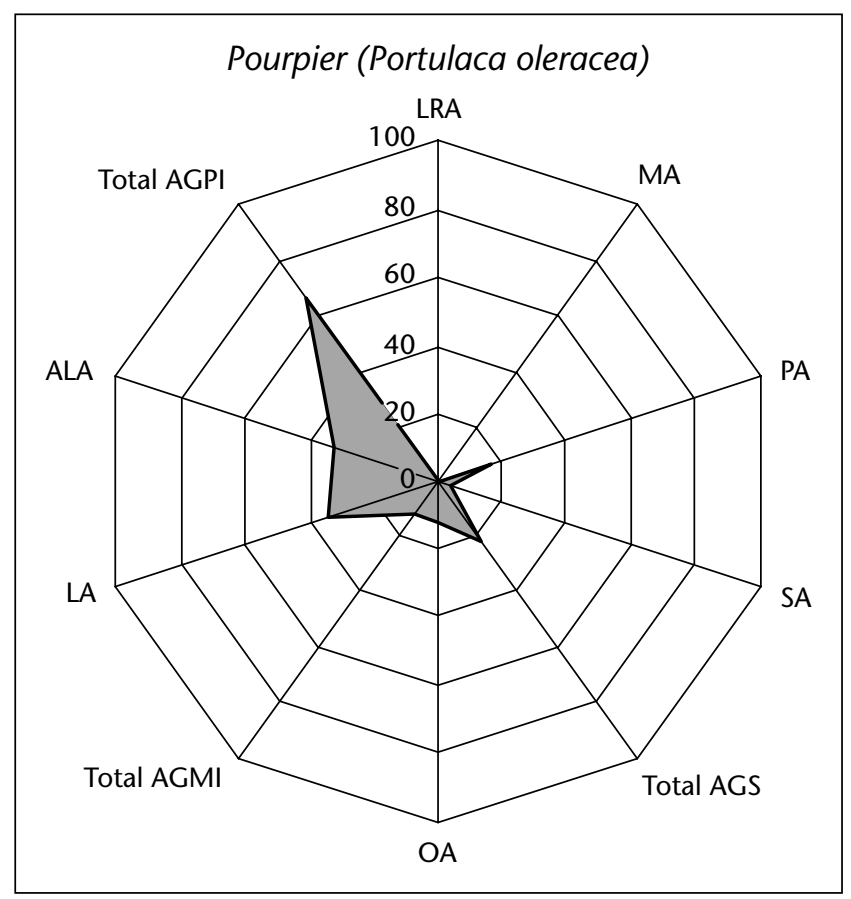

Figure 11. Profil en acides gras de I'huile de pourpier.

Les sources végétales classées selon leur profil en acides gras

Les tableaux 1 à 7 résument les données disponibles à propos des sources d'huiles, classées selon les clés présentées ci-dessus, c'est-à-dire selon leur profil en acides gras.

Les sources sont regroupées en fonction de leur type d'acides gras majoritaire (AGS, AGMI et AGPI). Chaque classe est subdivisée selon le deuxième acide gras majoritaire du profil. 


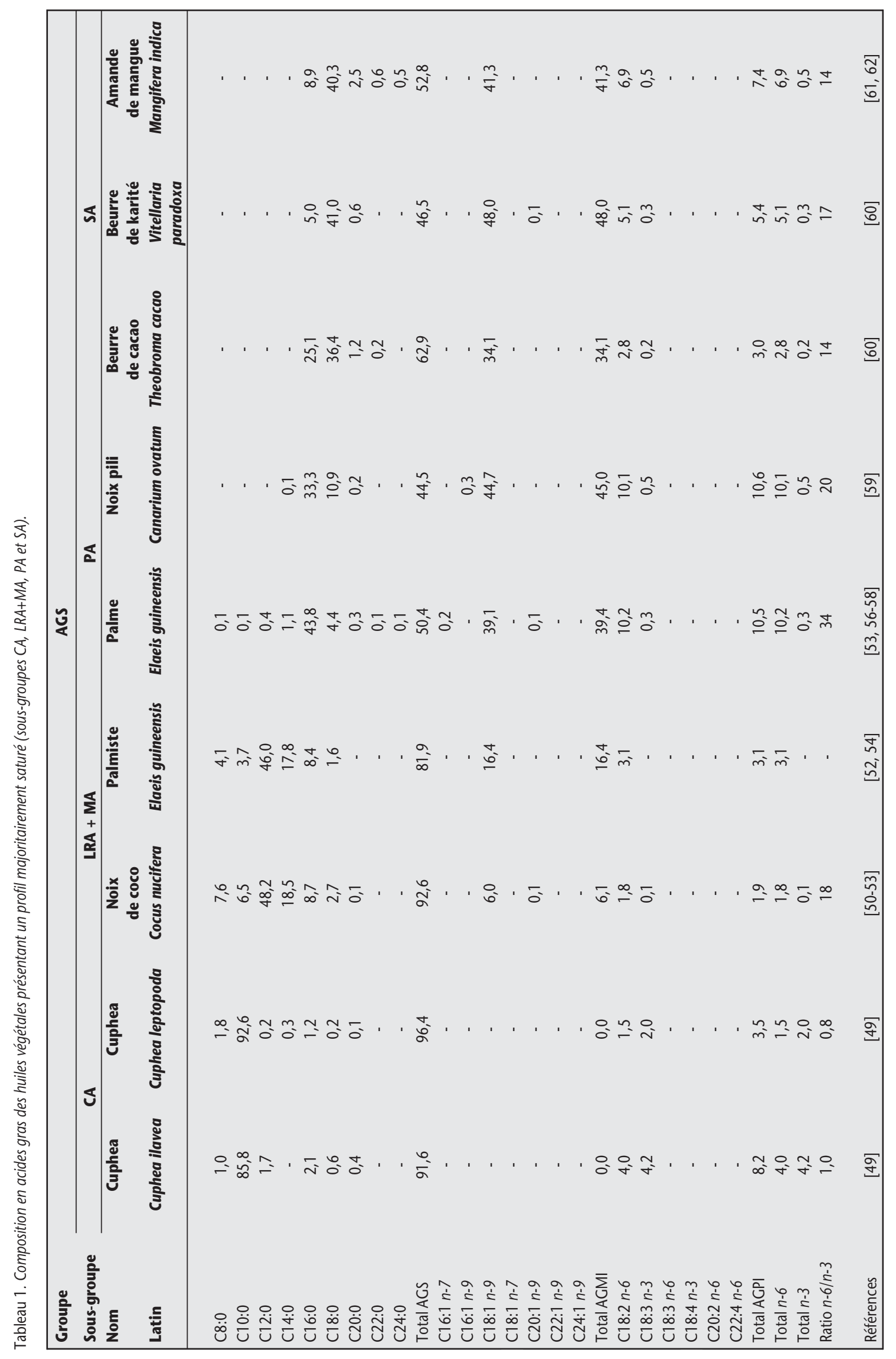




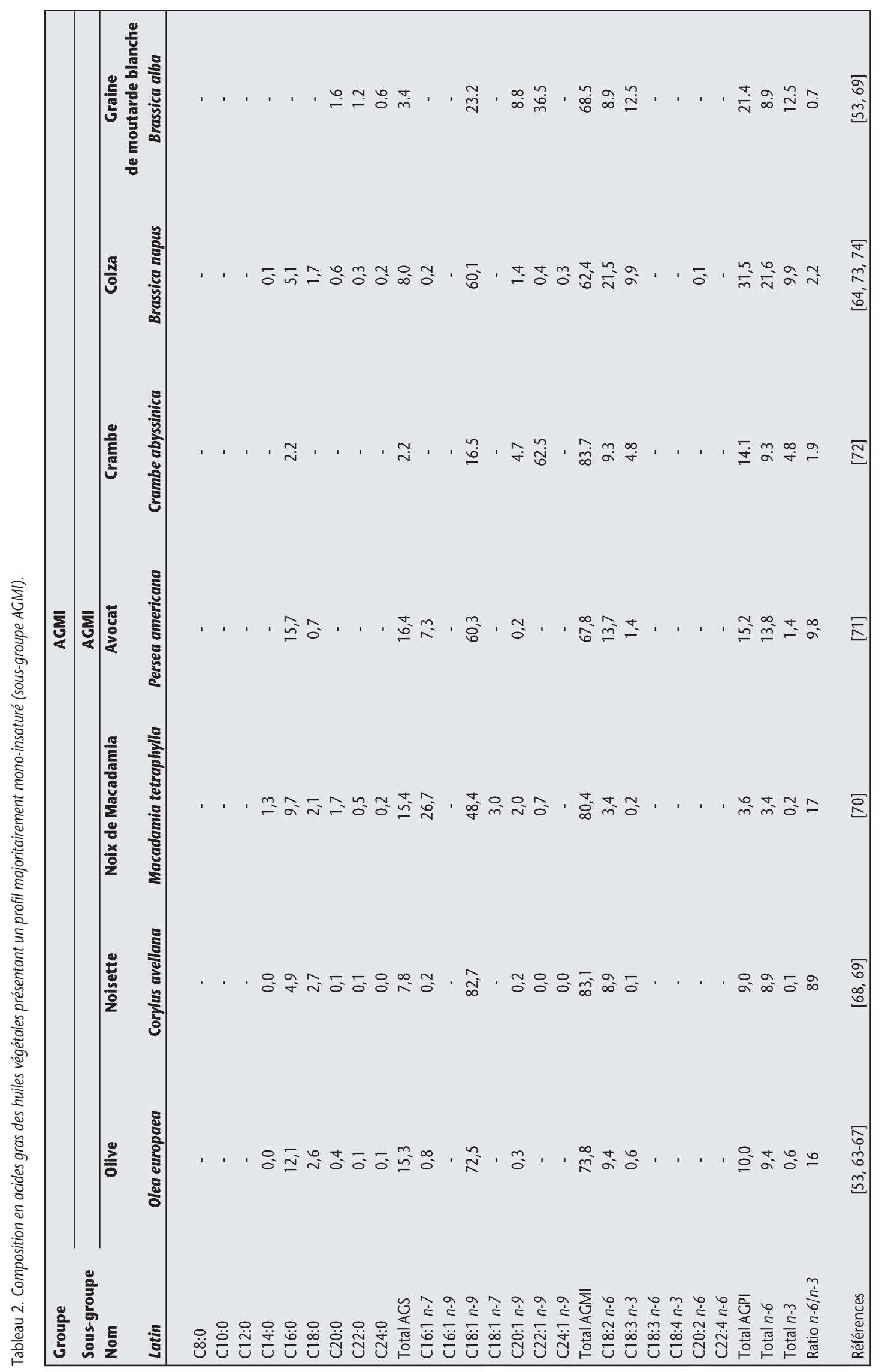




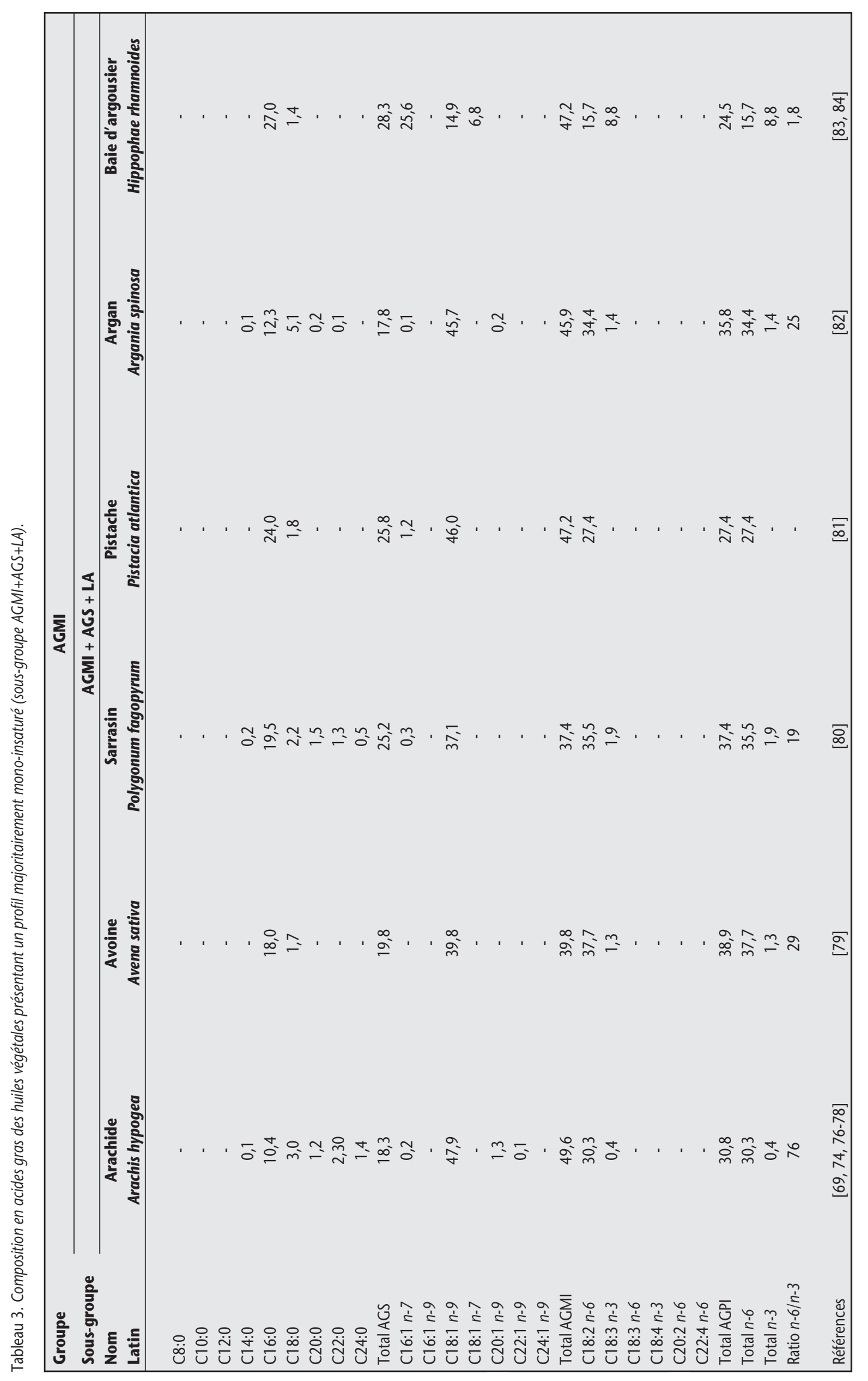




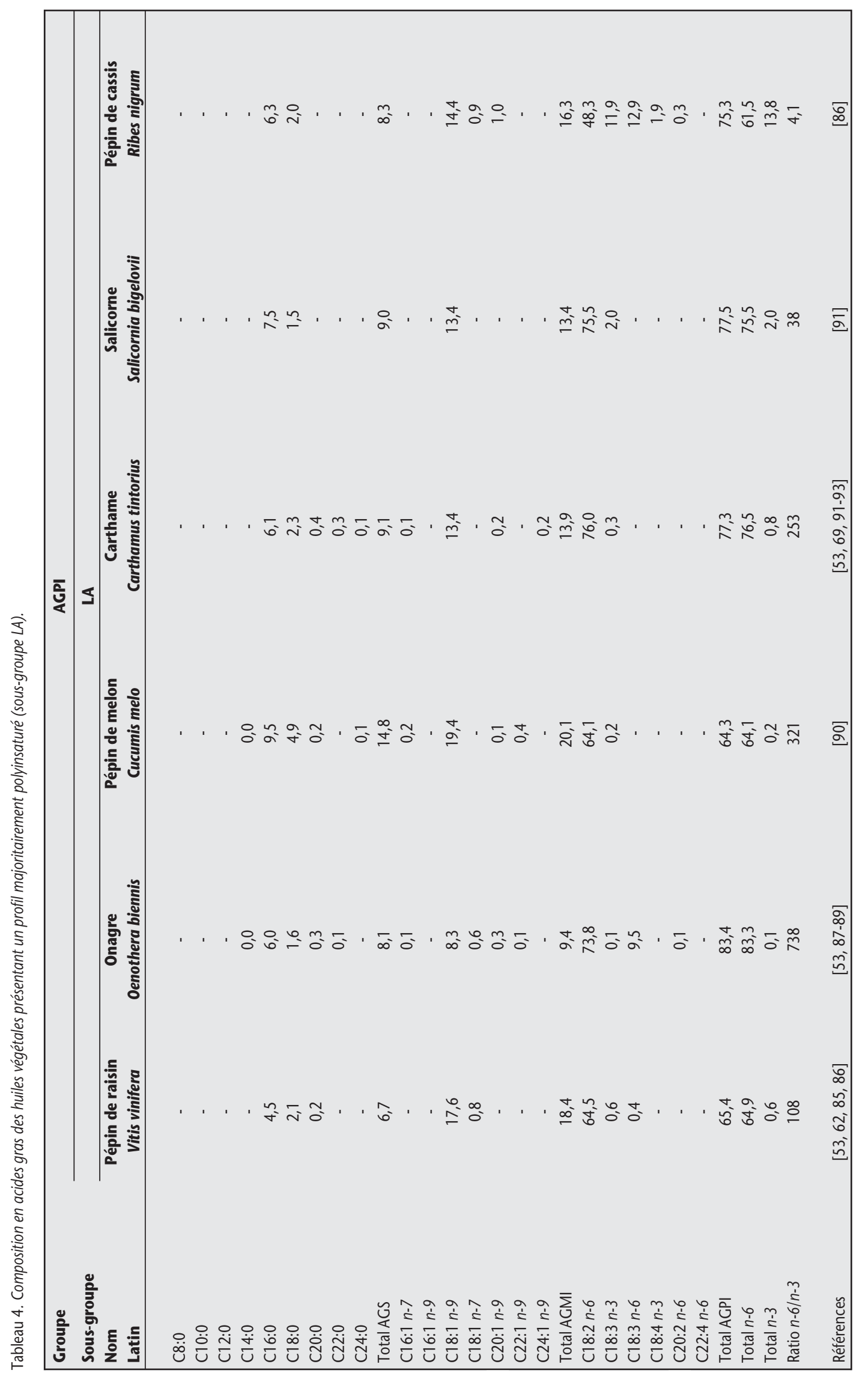




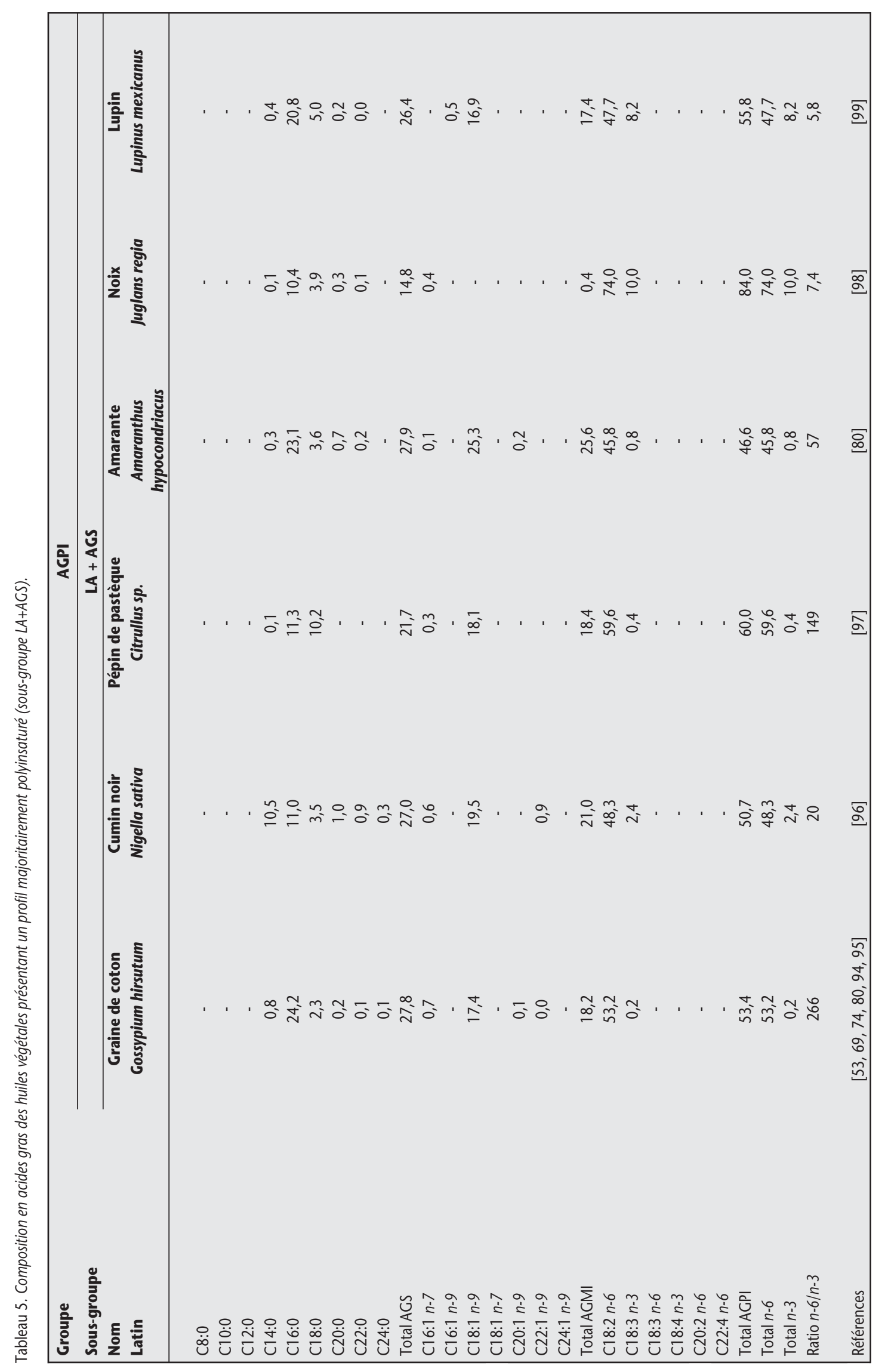

OCL VOL. 15 № 1 JANVIER-FÉVRIER $2008 \quad 67$ 


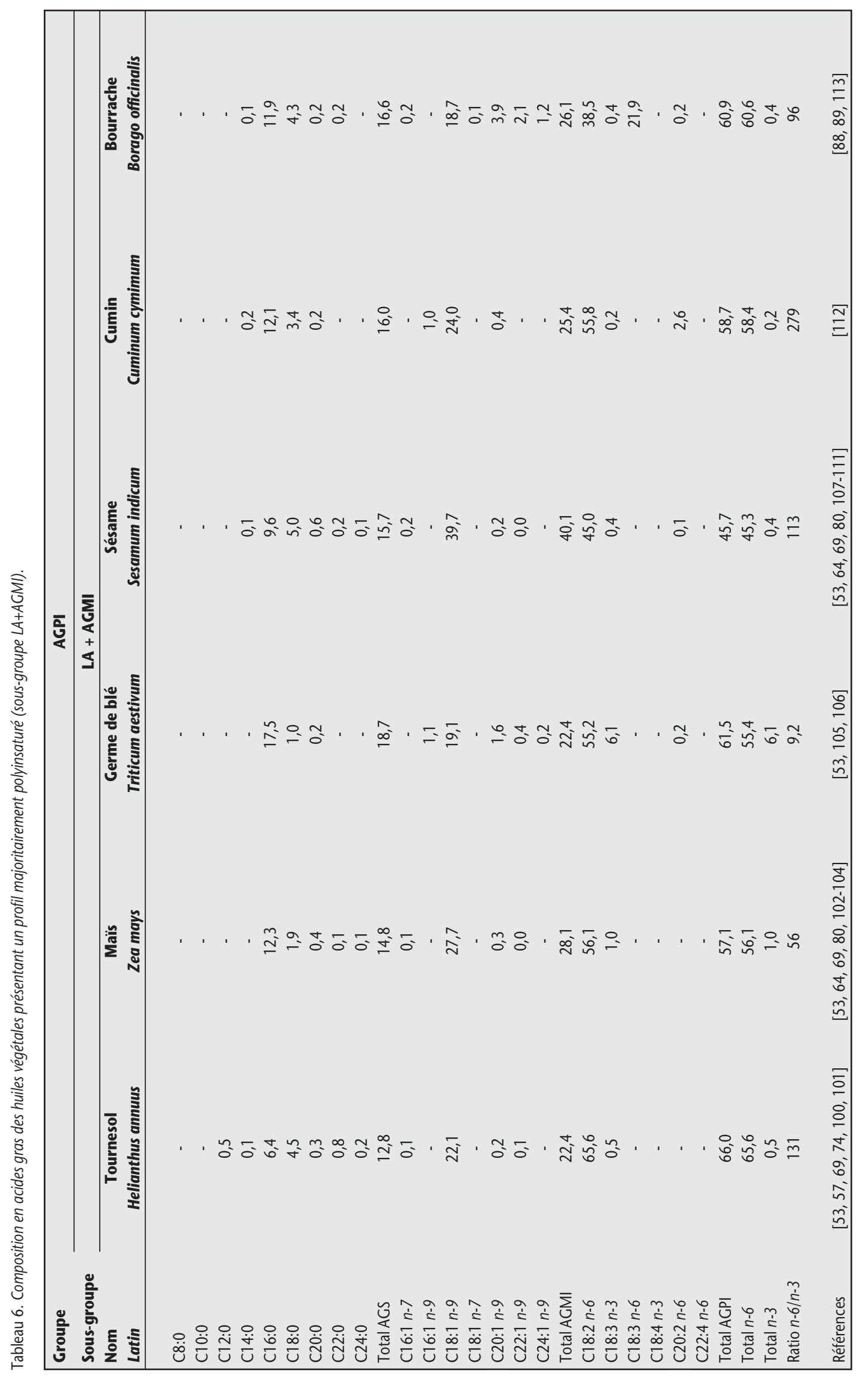




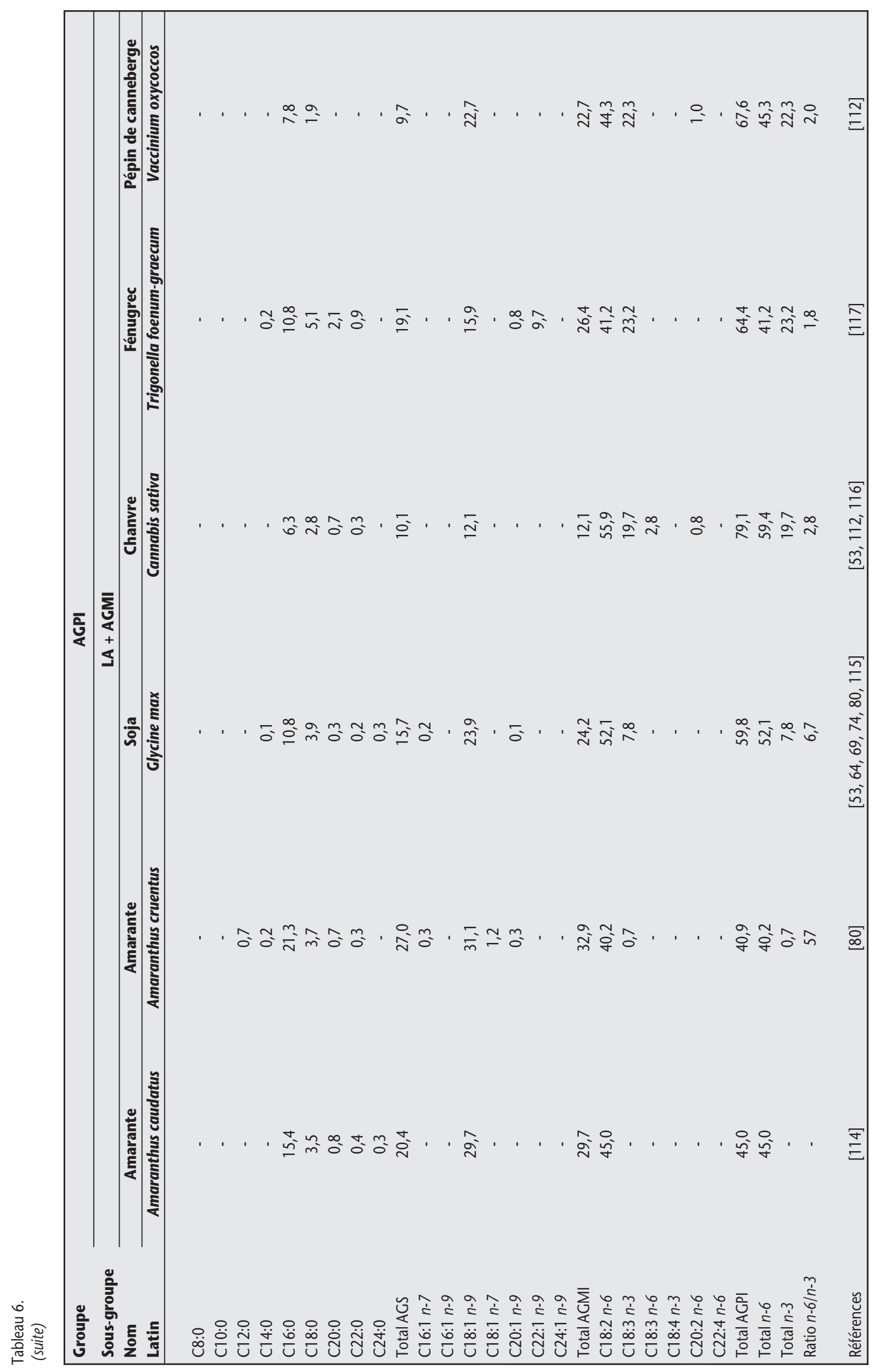




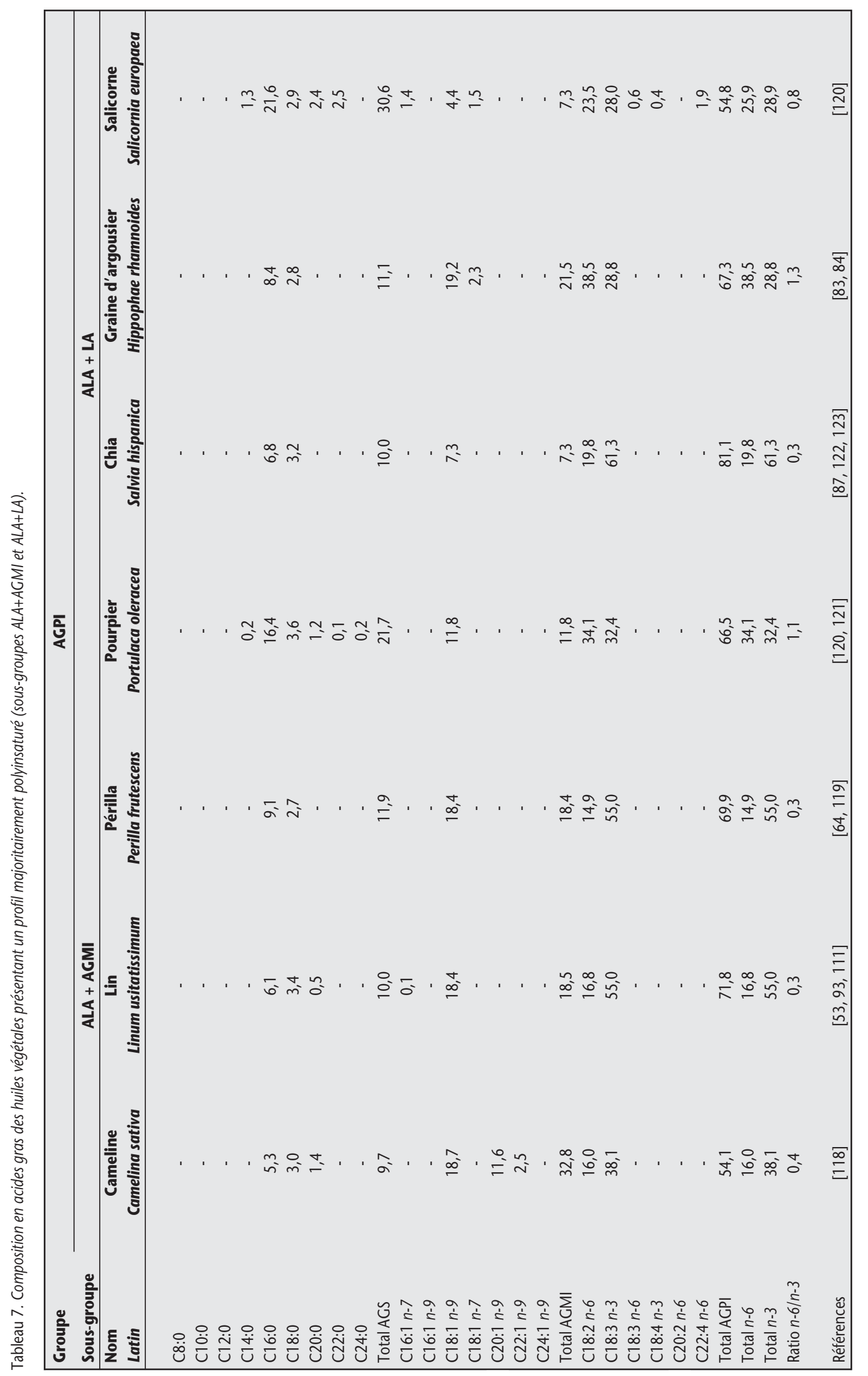

70 OCL VOL. 15 № 1 JANVIER-FÉVRIER 2008 
Cette classification permet d'avoir une image générale du potentiel nutritionnel de nombreuses sources d'huiles végétales, et constitue un outil intéressant pour le formulateur de produits à matrice lipidique.

\section{Discussion}

Dans le régime alimentaire occidental, comme par exemple en France, en Allemagne ou au Royaume-Uni, les lipides représentent $40 \%$ de la ration calorique, les AGS comptant pour la moitié. La proportion d'AGPI est inférieure à $15 \%(11 \%$ de LA, 0,8 \% d'ALA, 0,25\% d'EPA+DHA), avec un ratio $n-6 / n-3$ aux alentours de 15 [124-126]. Ceci ne correspond pas aux recommandations de différentes agences gouvernementales, qui conseillent en général un apport lipidique ne dépassant pas $30 \%$ de l'énergie, avec un maximum de $33 \%$ d'ACS et un ratio $n-6 / n-3$ de 5 ou moins : I'Agence française de sécurité sanitaire des aliments (AFSSA) [11], le National Cholesterol Education Program édité par un groupe de travail d'experts américains pour le management de l'hypercholestérolémie [127], I'American Heart Association [128] et la Deutsche Gesellschaft für Ernährung (équivalent allemand de l'AFSSA) [129].

La récente prise de conscience de l'industrie agroalimentaire a entraîné une évolution de la perception de l'apport lipidique par la prise en compte de la notion de " qualité des lipides ", en complément de celle de « quantité de lipides». Pour répondre à cette demande, en particulier en AGPI comme l'ALA, de nouvelles sources d'huiles végétales doivent être utilisées à la place des classiques huiles de tournesol ou de palme. La source majeure qui permet aux industriels de répondre à cette problématique à l'heure actuelle est l'huile de colza.

La classification réalisée dans cet article met en lumière deux groupes différents, selon les acides gras recherchés par le formulateur. Les huiles de l'avant-dernier groupe - cameline, lin et périlla - se distinguent par leur très forte teneur en ALA, si seul cet acide gras est nécessaire, et celles du dernier groupe - pourpier, chia, graine d'argousier et salicorne - sont intéressantes si les deux AGPI essentiels sont recherchés.

Plusieurs difficultés majeures peuvent empêcher l'utilisation de ces huiles nutritionnellement intéressantes. La première est le statut alimentaire de ces produits. En effet, l'huile de périlla a reçu un avis défavorable du Conseil supérieur d'hygiène publique de France (CSHPF) [130], que ce soit en alimentation courante ou en complément alimentaire. L'huile de lin a été interdite en alimentation en France [131, 132], mais un rapport récent de l'AFSSA a proposé un avis différent, qui doit encore être validé par la Direction générale de la concurrence, de la consommation et de la répression des fraudes (DGCCRF) [133]. Pour les autres huiles, la première entreprise qui voudra les utiliser dans un produit alimentaire devra remplir un dossier de "Novel Food" auprès de l'European Food Safety Authority (EFSA), ce qui représente un parcours long et coûteux.

Une deuxième difficulté est la disponibilité de ces huiles. En effet, si la cameline, le périlla, le lin et l'argousier existent en format industriel ou semi-industriel, ce n'est pas le cas pour les huiles de chia, de pourpier ou de salicorne. Une production industrielle de chia a été lancée en Argentine en 1998, mais il semble que des difficultés aient entravé le projet puisque cette matière première n'est toujours pas disponible sur le marché. Le pourpier est peut-être une piste intéressante, puisqu'il est utilisé en Grèce (en particulier en Crète) comme aliment pour le bétail. Enfin, il est important de noter que l'utilisation d'huiles polyinsaturées, comme celles contenant de grandes quantités d'ALA par exemple, influe de manière négative sur la stabilité et donc sur la durée de vie des produits alimentaires. En effet, l'oxydabilité des huiles augmentant avec le nombre d'insaturations, l'emploi d'huile de cameline ou de lin nécessite un travail de formulation des matrices alimentaires, avec utilisation d'antioxydants, voire d'autres technologies de protection.

Additionnées, ces difficultés ont limité l'utilisation de ces sources alternatives en formulation industrielle alimentaire. Si une tendance vers des profils en acides gras plus équilibrés est visible dans le secteur des corps gras « non beurre », la majorité des produits alimentaires à matrice lipidique ne montre rien de semblable. Dans ces derniers cas, le coût reste un facteur majeur en formulation, et conduit à I'utilisation de sources traditionnelles, fiables et bon marché. Toutefois, la formulation devenant un exercice de plus en plus difficile au fur et à mesure que les cahiers des charges nutritionnels se complexifient, des sources alternatives permettant d'apporter majoritairement tel ou tel composant recherché sans « diluer » les autres déjà présents deviendront nécessaires. La présente étude s'est limitée à des sources bien identifiées, ayant fait l'objet de travaux structurés et publiés. Elle a donc ses propres limites, alors que de nombreuses autres ressources existent, en particulier dans la sphère agro-climatique tropicale, dont l'étude est à peine ébauchée [134]. L'utilisation de ces ressources reste toutefois conditionnée par les impératifs industriels que sont la sécurité des approvisionnements, la garantie de qualité et de constance, et finalement le prix de revient.

\section{RÉFÉRENCES}

1. BONANOME A, GRUNDY SM. Effect of dietary stearic acid on plasma cholesterol and lipoprotein levels. N Engl / Med 1988 ; 318 : 1244-8.

2. CLARKE R, FROST C, COLLINS R, APPLEBY P, PETO R. Dietary lipids and blood cholesterol: quantitative meta-analysis of metabolic ward studies. BMJ $1997 ; 314: 112-7$

3. GRUNDY SM. Comparison of monounsaturated fatty acids and carbohydrates for lowering plasma cholesterol. N Engl J Med 1986 ; 314(12) : 745-8.

4. HUNTER JE. Studies on effects of dietary fatty acids as related to their position on triglycerides. Lipids $2001 ; 36$ : 655-68.

5. HUNTER KA, CROSBIE LC, WEIR A, MILLER G), DUTTA-ROY AK. A residential study comparing the effects of diets rich in stearic acid, oleic acid, and linoleic acid on fasting blood lipids, hemostatic variables and platelets in young healthy men. J Nutr Biochem 2000 ; 11 : 408-16.

6. KITTS DD, JONES PJH. Dietary fats : discriminative partitioning for energy and synthesis of triacylglycerides. Food Res Int $1996 ; 29(1)$ : 57-69.

7. KRIS-ETHERTON PM, YU S. Individual fatty acid effects on plasma lipids and lipoproteins: human studies. Am J Clin Nutr 1997; 65(suppl): 1628S1644 S.

8. KUBOW S. The influence of positional distribution of fatty acids in native, interesterified and structure-specific lipids on lipoprotein metabolism and atherogenesis. / Nutr Biochem 1996 ; 7 : 530-41.

9. NAUDET M, SOULIER J, FARINES M. Principaux constituants chimiques des corps gras. In : Karleskind A, ed. Manuel des corps gras - Volume 1. Paris : Lavoisier Tec \& Doc, 1992 : 65-115.

10. KLERE J, BARSACQ JC, UZZAN A, KARLESKIND A, HOCHARD JM. Généralités. In : Karleskind A, ed. Manuel des corps gras - Volume 1. Paris : Lavoisier Tec \& Doc, 1992 : 1-64.

11. LEGRAND P, BOURRE JM, DESCOMPS B, DURAND G, RENAUD S. Lipides. In : Martin A, ed. Apports nutritionnels conseillés pour la population française. Paris : Lavoisier Tec \& Doc, 2001 : 63-82.

12. CULLEN $P$. Evidence that triglycerides are an independent coronary heart disease risk factor. Am / Cardiol 2000 ; 86 : 943-9.

13. DESPRES JP, LEMIEUX I, DAGENAIS GR, CANTIN B, LAMARCHE B. HDLcholesterol as a marker of coronary heart disease risk : the Quebec cardiovascular study. Atherosclerosis $2000 ; 153: 263-72$.

14. EBERLY LE, STAMLER J, NEATON JD, Multiple Risk Factor Intervention Trial Research Group. Relation of triglyceride levels, fasting and nonfasting, to fatal and nonfatal coronary heart disease. Arch Intern Med 2003 ; 163(9) : 1077-83. 
15. FRISHMAN WH. Biologic markers as predictors of cardiovascular disease. Am J Med $1998 ;$ 104(6A) : 18S-27S.

16. JEPPESEN J, HEIN HO, SUADICANI P, GYNTELBERG F. Relation of high TG-low HDL cholesterol and LDL cholesterol to the incidence of ischemic heart disease. Arterioscler Thromb Vasc Biol 1997 ; 17 : 1114-20.

17. KRIS-ETHERTON PM, HECKER KD, BINKOSKI AE. Polyunsaturated fatty acids and cardiovascular health. Nutr Rev $2004 ; 62(11):$ 414-26.

18. ROCHE HM, GIBNEY MJ. Effect of long chain $n-3$ polyunsaturated fatty acids on fasting and postprandial triacylglycerol metabolism. Am / Clin Nutr 2000 ; 71 (suppl) : 232S-237S.

19. SPRECHER DL, WATKINS TR, BEHAR S, BROWN WV, RUBINS HB, SCHAEFE$R$ EJ. Importance of high-density lipoprotein cholesterol and triglyceride levels in coronary heart disease. Am / Cardiol $2003 ; 91$ : 575-80.

20. ABEYWARDENA MY, HEAD RJ. Long chain $n-3$ polyunsaturated fatty acids and blood vessel function. Cardiovasc Res $2001 ; 52$ : 361-71.

21. ABEYWARDENA MY, MC LENNAN PL, CHARNOCK IS. Differential effects of dietary fish oil on myocardial prostaglandin $\mathrm{I}_{2}$ and thromboxane $\mathrm{A}_{2}$ production. Am J Physiol $1992 ; 260$ : H379-H385.

22. LEHR HA, HUBNER C, FINCKHB, ETAL. Dietary fish oil reduces leukocyte/endothelium interaction following systemic administration of oxidatively modified low density lipoprotein. Circulation 1991 ; 84 : 1725-31.

23. GRUNDY SM, DENKE MA. Dietary influences on serum lipids and lipoproteins. J Lipid Res $1990 ; 31$ : 1149-72.

24. KHOSLA P, SUNDRAM K. Effects of dietary fatty acid composition on plasma cholesterol. Prog Lipid Res 1996 ; 35(2) : 93-132.

25. POISSON JP, NARCE M. Corps gras alimentaires: aspects chimiques et biochimiques. In : Graille J, ed. Lipides et corps gras alimentaires. Paris : Lavoisier Tec \& Doc, 2003 : 1-50.

26. DABADIE H, PEUCHANT E, BERNARD M, LERUYET P, MENDY F. Moderate intake of myristic acid in sn-2 position has beneficial lipidic effects and enhances DHA of cholesteryl esters in an interventional study. J Nutr Biochem $2005 ; 16: 375-82$

27. HAYES KC, KHOSLA P. Dietary fatty acid thresholds and cholesterolemia. FASEB / $1992 ; 6: 2600-7$

28. DOTSON KD, JERRELL JP, PICCIANO MF, PERKINS EG. High-performance liquid chromatography of human milk triacylglycerols and gas chromatography of component fatty acids. Lipids $1992 ; 27: 933-9$.

29. BENNETT CLARK S, DERKSEN A, SMALL DM. Plasma clearance of emulsified triolein in conscious rats : effects of phosphatidylcholine species, cholesterol content and emulsion surface physical state. Exp Physiol 1991 ; 76 : 39-52.

30. HARRIS WS. Fish oils and plasma lipids and lipoprotein metabolism in humans : a critical review. J Lipid Res $1989 ; 30$ : 785-807.

31. HARRIS WS. $n-3$ Fatty acids and serum lipoproteins : human studies. Am I Clin Nutr $1997 ;$; 65(suppl) : 1645S-1654S.

32. WIJENDRAN V, HAYES KC. Dietary $n-6$ and $n-3$ fatty acid balance and cardiovascular health. Annu Rev Nutr 2004 ; 24 : 597-615.

33. JUDD JT, BAER DJ, CLEVIDENCE BA, ET AL. Effects of margarine compared with those of butter on blood lipid profiles related to cardiovascular disease risk factors in normolipemic adults fed controlled diets. Am / Clin Nutr 1998 ; $68: 768-77$.

34. MATTSON FH, GRUNDY SM. Comparison of effects of dietary saturated, monounsaturated and polyunsaturated fatty acids on plasma lipids and lipoproteins in man. / Lipid Res 1985 ; 26 : 194-202.

35. WOOD R, KUBENA K, O'BRIEN B, TSENG S, MARTIN G. Effect of butter, mono and polyunsaturated fatty acid-enriched butter, trans fatty acid margarine, and zero trans fatty acid margarine on serum lipids and lipoproteins in healthy men. / Lipid Res $1993 ; 34$ : 1-11.
36. HORROBIN DF, HUANG YS. The role of linoleic acid and its metabolites in the lowering of plasma cholesterol and the prevention of cardiovascular disease. Int / Cardiol 1987 ; 17 : 241-55.

37. LASSERRE M, MENDY F, SPIELMANN D, JACOTOT B. Effects of different dietary intake of essential fatty acids on $C 20: 3$ omega 6 and $C 20: 4$ omega6 serum levels in human adults. Lipids $1985 ; 20(4)$ : 227-33.

38. DE LORGERIL M, SALEN P, MARTIN JL, MONJAUD I, DELAYE J, MAMELLE N. Mediterranean diet, traditional risk factors, and the rate of cardiovascular complications after myocardial infarction. Circulation $1999 ; 99$ : 779-85.

39. FINNEGAN YE, MINIHANE AM, LEIGH-FIRBANK EC, ET AL. Plant and marine derived $n-3$ polyunsaturated fatty acids have differential effects on fasting and postprandial blood lipid concentrations and on the susceptibility of LDL to oxidative modification in moderately hyperlipidemic subjects. Am / Clin Nutr $2003 ; 77$ : 783-95.

40. OOMEN CM, OCKE MC, FESKENS EJM, KOK FJ, KROMHOUT D. Alphalinolenic acid intake is not beneficially associated with 10 -y risk of coronary artery disease incidence : the Zutphen Elderly Study. Am / Clin Nutr 2001 ; 74 : 457-63.

41. SINGH RB, NIAZ MA, SHARMA JP, KUMAR R, RASTOGI V, MOSHIRI M. Randomised, double-blind, placebo-controlled trial of fish oil and mustard oil in patients with suspected acute myocardial infarction : the Indian Experiment of Infarct Survival-4. Cardiovasc Drugs Ther 1997 ; 11 : 485-91.

42. ROS E, NUNEZ I, PEREZ-HERAS A, ET AL. A walnut diet improves endothelial function in hypercholesterolemic subjects. Circulation $2004 ; 109$ : 1609-14.

43. INDU M, GHAFOORUNISSA. $n-3$ Fatty acids in Indian diets. Comparison of the effects of precursor ( $\alpha$-linolenic acid) vs. product (long chain $n-3$ polyunsaturated fatty acids). Nutr Res $1992 ; 12: 569-82$.

44. WILKINSON P, LEACH C, AH-SING EE, ET AL. Influence of $\alpha$-linolenic acid and fish oil on markers of cardiovascular risk in subjects with an atherogenic lipoprotein phenotype. Atherosclerosis $2005 ; 181(1)$ : 115-24.

45. ZHAO G, ETHERTON TD, MARTIN KR, WEST SG, GILLIES PJ, KRISETHERTON PM. Dietary a-linolenic acid reduces inflammatory and lipid cardiovascular risk factors in hypercholesterolemic men and women. I Nutr $2004 ; 134$ : 2991-7.

46. SINGH RB, DUBNOV G, NIAZ MA, ET AL. Effect of an Indo-Mediterranean diet on progression of coronary artery disease in high risk patients (IndoMediterranean Diet Heart Study) : a randomised single-blind trial. Lancet $2002 ; 360: 1455-61$.

47. SINGH RB, RASTOGISS, VERMA R, ET AL. Randomised controlled trial of cardioprotective diet in patients with recent acute myocardial infarction : results of one year follow up. BMJ $1992 ; 304: 1015-9$.

48. SANDERSON P, FINNEGAN YE, WILLIAMS CM, ET AL. UK food standards agency $\alpha$-linolenic acid workshop report. Br J Nutr 2002 ; 88 : 573-9.

49. SAIKUSA T, KITTA K, OHKAWA Y, FUJII Y, KOUZAI S, MORI Y. EDIBLE PROPERTIES OF CUPHEA SEED OIL. Nippon Shokuhin Kagaku Kogaku Kaishi $2001 ; 48(3): 210-3$.

50. CANAPI EC, AGUSTIN YTV, MORO EA, PEDROSA JR. E, BENDANO MLJ. Coconut oil. In : Hui YD, ed. Bailey's industrial oil and fat products - Edible oil and fat products : oil and oilseeds. New York : John Wiley and Sons Inc., 1996 : 97-124.

51. LAURELES LR, RODRIGUEZ FM, REANO CE, ET AL. Variability in fatty acid and triacylglycerol composition of the oil of coconut (Cocos nucifera L.) hybrids and their parentals. J Agric Food Chem 2002 ; 50 : 1581-6.

52. PANTZARIS TP, BASIRON Y. The lauric oils. In : Gunstone FD, ed. Vegetable oils in food technology, composition, properties and uses. Oxford : Blackwell Publishing Ltd., 2002 : 157-202.

53. YOUDIM KA, MARTIN A, JOSEPH JA. Essential fatty acids and the brain : possible health implications. Int I Dev Neurosci $2000 ; 18: 383-99$. 
54. BEREAU D, BENJELLOUN-MLAYAH B, BANOUB J, BRAVO R. FA and unsaponifiable composition of five Amazonian palm kernel oils. I Am Oil Chem Soc $2003 ; 80(1): 49-53$.

55. RATNAYAKE WMN, DAUN JK. Chemical composition of canola and rapeseed oils. In : Gunstone FD, ed. Vegetable oils in food technology, composition, properties and uses. Oxford: Blackwell Publishing Ltd., 2002: 37-78.

56. LIN SW. PALM OIL. IN : GUNSTONE FD, ED. Vegetable oils in food technology, composition, properties and uses. Oxford: Blackwell Publishing Ltd., 2002 : 59-97.

57. NOOR LIDA HMD, SUNDRAM K, SIEW WL, AMINAH A, MAMOT S. TAG composition and solid fat content of palm oil, sunflower oil, and palm kernel olein blends before and after chemical interesterification. J Am Oil Chem Soc $2002 ; 79(11)$ : 1137-44.

58. RAJANAIDU N, KUSHARI A, RAFII A, MOHD DIN A, PAIZURA I, JALANI BS Oil palm breeding and genetic resources. In : Yusof B, Jalani BS, Chan KW, eds. Advances in oil palm research. Malaysia : Malaysian Palm Oil Board, $2000: 171-224$.

59. KAKUDA Y, JAHANIAVAL F, MARCONE MF, MONTEVIRGEN L, MONTEVIRGEN Q, UMALI J. Characterization of pili nut (Canarium ovatum) oil : fatty acid and triacylglycerol composition and physicochemical properties. / Am Oil Chem Soc $2000 ; 77(9)$ : 991-6.

60. LIPP M, ANKLAM E. Review of cocoa butter and alternative fats for use in chocolate. Part A : Compositional data. Food Chem 1998 ; 62(1) : 73-97.

61. SOLIS-FUENTES JA. DURAN-DE-BAZUA MC. Mango seed uses: thermal behaviour of mango seed almond fat and its mixture with cocoa butter. Bioresour Technol $2004 ; 92$ : 71-8.

62. UDAYASEKHARA RAO P. Nutrient composition of some less-familiar oil seeds. Food Chem $1994 ; 50: 379-82$.

63. BOSKOU D. OLIVE OIL. In : Gunstone FD, ed. Vegetable oils in food technology, composition, properties and uses. Oxford: Blackwell Publishing Ltd., 2002 : 244-77.

64. LEE DS, NOH BS, BAE SY, KIM K. Characterization of fatty acids composition in vegetable oils by gas chromatography and chemometrics. Anal Chim Acta $1998 ; 358: 163-75$.

65. MANNINA L, DUGO G, SALVO F, ET AL. Study of the cultivar-composition relationship in Sicilian olive oils by GC, NMR, and statistical methods. / Agric Food Chem 2003 ; 51 : 120-7.

66. OLLIVIER D, ARTAUD J, PINATEL C, DURBEC JP, GUERERE M. Triacylglycerol and fatty acid compositions of French virgin olive oils, characterization by chemometrics. J Agric Food Chem 2003 ; 51 : 5723-31.

67. RANALLI A, POLLASTRI L, CONTENTO S, ET AL. Acylglycerol and fatty acid components of pulp, seed and whole olive fruit oils : Their use to characterize fruit variety by chemometrics. J Agric Food Chem $2002 ; 50$ : 3775-9.

68. ALASALVAR C, SHAHIDI F, OHSHIMA T, ET AL. Turkish Tombul hazelnut (Corylus avellana L.). 2. Lipid characteristics and oxidative stability. / Agric Food Chem $2003 ; 51: 3797-805$

69. CHRISTOPOULOU E, LAZARAKI M, KOMAITIS M, KASELIMIS K. Effectiveness of determinations of fatty acids and triglycerides for the detection of adulteration of olive oils with vegetable oils. Food Chem 2004 ; 84 : 463-74

70. KAIJSER A, DUTTA P, SAVAGE G. Oxidative stability and lipid composition of macadamia nuts grown in New Zealand. Food Chem $2000 ; 71: 67-70$.

71. MORENO AO, DORANTES L, GALINDEZ I, GUZMAN RI. Effect of different extraction methods on fatty acids, volatile compounds, and physical and chemical properties of avocado (Persea americana Mill.) oil. J Agric Food Chem $2003 ; 51: 2216-21$.

72. WANG YP, TANG JS, CHU CQ, TIAN J. A preliminary study on the introduction and cultivation of Crambe abyssinica in China, an oil plant for industrial uses. Ind Crops Prod $2000 ; 12$ : 47-52.
73. PRZYBYLSKI R, MAG T. Canola Rapeseed oil. In : Gunstone FD, ed. Vegetable oils in food technology, composition, properties and uses. Oxford: Blackwell Publishing Ltd., 2002 : 98-127.

74. WANG T. Soybean oil. In : Gunstone FD, ed. Vegetable oils in food technology, composition, properties and uses. Oxford: Blackwell Publishing Ltd., $2002: 18-58$.

75. THOMASSON HJ, BOLDINGH J. The biological value of oils and fats. II. The growth-retarding substance in rapeseed oil. J Nutr 1955 ; 56(4) : 469-75.

76. DORSCHEL CA. Characterization of the TAG of peanut oil by electrospray LC-MS-MS. J Am Oil Chem Soc 2002 ; 79(8) : 749-53.

77. SANDERS TH. Groundnut (peanut) oil. In : Gunstone FD, ed. Vegetable oils in food technology, composition, properties and uses. Oxford: Blackwell Publishing Ltd., 2002 : 231-43.

78. YOUNG CT. Peanut oil. In : Hui YD, ed. Bailey's industrial oil and fat products - Edible oil and fat products : oil and oilseeds. New York : John Wiley and Sons Inc., 1996 : 377-92.

79. ZHOU MX, HOLMES MG, ROBARDS K, HELLIWELL S. Fatty acid composition of lipids of Australian oats. J Cereal Sci 1998 ; 28 : 311-9.

80. JAHANIAVAL F, KAKUDA Y, MARCONE MF. Fatty acid and triacylglycerol compositions of seed oils of five Amaranthus accessions and their comparison to other oils. J Am Oil Chem Soc 2000 ; 77(8) : 847-52.

81. YOUSFI M, NEDJMI B, BELLAL R, BEN BERTAL D, PALLA G. Fatty acids and sterols of Pistacia atlantica fruit oil. J Am Oil Chem Soc 2002; 79(10): 1049-50.

82. REZANKA T, REZANKOVA H. Characterization of fatty acids and triacylglycerols in vegetable oils by gas chromatography and statistical analysis. Anal Chim Acta 1999 ; 398 : 253-61.

83. KALLIO H, YANG B, PEIPPO P, TAHVONEN R, PAN R. Triacylglycerols, glycerophospholipids, tocopherols and tocotrienols in berries and seeds of two subspecies (ssp. sinensis and mongolica) of sea buckthorn (Hippophaë rhamnoides). J Agric Food Chem $2002 ; 50$ : 3004-9.

84. YANG B, KALLIO HP. Fatty acid composition of lipids in sea buckthorn (Hippophä̈ rhamnoides L.) berries of different origins. J Agric Food Chem $2001 ; 49$ : 1939-47.

85. CAO X, ITO Y. Supercritical fluid extraction of grape seed oil and subsequent separation of free fatty acids by high-speed counter-current chromatography. J Chromatogr 2003 ; 1021 : 117-24.

86. SOVOVA H, ZAREVUCKA M, VACEK M, STRANSKY K. Solubility of two vegetable oils in supercritical $\mathrm{CO}_{2}$. / Supercrit Fluids $2001 ; 20: 15-28$.

87. HEUER B, YANIV Z, RAVINA I. Effect of late salinization of chia (Salvia hispanica), stock (Matthiola tricuspidata) and evening primrose (Oenothera biennis) on their oil content and quality. Ind Crops Prod 2002 ; 15 : 163-7.

88. REDDEN PR, HUANG YS, LIN X, HORROBIN DF. Separation and quantification of the triacylglycerols in evening primrose and borage oils by reversedphase high-performance liquid chromatography. J Chromatogr 1995 ; 694 : $381-9$.

89. SENANAYAKE SPJN, SHAHIDI F. Chemical and stability characteristics of structured lipids from borage (Borago officinalis L.) and evening primrose (Oenothera biennis L.) oils. J Food Sci 2002 ; 67(6) : 2038-45.

90. DE MELO MLS, NARAIN N, BORA PS. Characterization of some nutritional constituents of melon (Cucumis melo hybrid AF-522) seeds. Food Chem $2000 ; 68: 411-4$

91. ANWAR F, BHANGER MI, NASIR MKA, ISMAIL S. Analytical characterization of Salicornia bigelovii seed oil cultivated in Pakistan. J Agric Food Chem 2002 ; $50: 4210-4$.

92. OOMAH BD, LADET S, GODFREY DV, LIANG J, GIRARD B. Characteristics of raspberry (Rubus idaeus L.) seed oil. Food Chem 2000 ; 69 : 187-93. 
93. RALLIDIS LS, PASCHOS G, LIAKOS GK, VELISSARIDOU H, ANASTASIADIS G ZAMPELAS A. Dietary $\alpha$-linolenic acid decreases $C$-reactive protein, serum amyloid A and interleukin-6 in dyslipidemic patients. Atherosclerosis 2003 ; $167: 237-42$

94. JONES LA, KING CC. Cottonseed oil. In : Hui YD, ed. Bailey's industrial oil and fat products - Edible oil and fat products : oil and oilseeds. New York : John Wiley and Sons Inc., $1996: 159-240$.

95. O'BRIEN RD. Cottonseed oil. In : Gunstone FD, ed. Vegetable oils in food technology, composition, properties and uses. Oxford: Blackwell Publishing Ltd., 2002 : 203-30.

96. ATTA MB. Some characteristics of nigella (Nigella sativa L.) seed cultivated in Egypt and its lipid profile. Food Chem $2003 ; 83: 63-8$.

97. EL-ADAWY TA, TAHA KM. Characteristics and composition of watermelon, pumpkin, and paprika seed oils and flours. J Agric Food Chem 2001 ; 49 : 1253-9.

98. TSAMOURIS G, HATZIANTONIOU S, DEMETZOS C. Lipid analysis of Greek walnut oil (Juglans regia L.). Z Naturforsch 2002 ; 57c : 51-6.

99. GARCIA-LOPEZ PM, MUZQUIZ M, RUIZ-LOPEZ MA, ET AL. Chemical composition and fatty acid profile of several Mexican wild lupins. J Food Compost Anal $2001 ; 14: 645-51$.

100. GUINDA A, DOBARGANES MC, RUIZ-MENDEZ MV, MANCHA M. Chemical and physical properties of a sunflower oil with high levels of oleic and palmitic acids. Eur J Lipid Sci Technol 2003 ; 105 : 130-7.

101. GUPTA MK. Sunflower oil. In: Gunstone FD, ed. Vegetable oils in food technology, composition, properties and uses. Oxford: Blackwell Publishing Ltd., 2002 : 128-56.

102. GOFFMAN FD, BOHME T. Relationship between fatty acid profile and vitamin E content in maize hybrids (Zea mays L.). J Agric Food Chem 2001 ; 49 : 4990-4.

103. MOREAU RA. Corn oil. In : Gunstone FD, ed. Vegetable oils in food technology, composition, properties and uses. Oxford: Blackwell Publishing Ltd., 2002 : 278-96.

104. STRECKER LR, BIEBER MA, MAZA A, GROSSBERGER T, DOSKOCZYNSKI W]. Corn oil. In : Hui YD, ed. Bailey's industrial oil and fat products - Edible oil and fat products : oil and oilseeds. New York : John Wiley and Sons Inc., 1996 : 125-58.

105. DUNFORD NT, ZHANG M. Pressurized solvent extraction of wheat germ oil. Food Res Int $2003 ; 36: 905-9$.

106. PANFILI G, CINQUATA L, FRATIANNI A, CUBADDA R. Extraction of wheat germ oil by supercritical $\mathrm{CO}_{2}$ : Oil and defatted cake characterization. / Am Oil Chem Soc 2003 ; 80(2) : 157-61.

107. ABOU-GHARBIA HA, SHEHATA AAY, SHAHIDI F. Effect of processing on oxidative stability and lipid classes of sesame oil. Food Res Int $2000 ; 33: 331-40$.

108. ALPALSAN M, BOYDAK E, HAYTA M, GERCEK S, SIMSEK M. Effect of row space and irrigation on seed composition of Turkish sesame (Sesamum indicum L.). J Am Oil Chem Soc 2001 ; 78(9) : 933-5.

109. CHUNG CH, YEE Y], KIM DH, KIM HK, CHUNG DS. Changes of lipid, protein, RNA and fatty acid composition in developing sesame (Sesamum indicum L.) seeds. Plant Sci 1995 ; 109 : 237-43.

110. DESHPANDE SS, DESHPANDE US, SALUNKHE DK. Sesame oil. In : Hui YD, ed. Bailey's industrial oil and fat products - Edible oil and fat products : oil and oilseeds. New York : John Wiley and Sons Inc., 1996 : 457-96.

111. KOCHHAR SP. Sesame, rice-bran and flaxseed oils. In : Gunstone FD, ed. Vegetable oils in food technology, composition, properties and uses. Oxford : Blackwell Publishing Ltd., 2002 : 297-326.
112. PARKER TD, ADAMS DA, ZHOU K, HARRIS M, YU L. Fatty acid composition and oxidative stability of cold-pressed edible seed oils. I Food Sci 2003 ; 68(4) : 1240-3.

113. MOLERO GOMEZ A, MARTINEZ DE LA OSSA E. Quality of borage seed oil extracted by liquid and supercritical carbon dioxide. Chem Eng / 2002 ; 88 : 103-9.

114. BRUNI R, MEDICI A, GUERRINI A, ET AL. Wild Amaranthus caudatus seed oil, a nutraceutical resource from Ecuadorian flora. J Agric Food Chem $2001 ; 49$ : 5455-60.

115. SIPOS EF, SZUHAJ BF. Soybean oil. In : Hui YD, ed. Bailey's industrial oil and fat products - Edible oil and fat products : oil and oilseeds. New York : John Wiley and Sons Inc., 1996 : 497-602.

116. OOMAH BD, BUSSON M, GODFREY DV, DROVER JCG. Characteristics of hemp (Cannabis sativa L.) seed oil. Food Chem 2002 ; 76 : 33-43.

117. EL-SEBAIY LA, EL-MAHDY AR. Lipid changes during germination of fenugreek seeds (Trigonella foenum-graecum). Food Chem $1983 ; 10:$ 309-19.

118. SHUKLA VKS, DUTTA PC, ARTZ WE. Camelina oil and its unusual cholesterol content. J Am Oil Chem Soc $2002 ; 79(10)$ : 965-9.

119. LONGVAH T, DEOSTHALE YG, KUMAR PU. Nutritional and short term toxicological evaluation of perilla seed oil. Food Chem $2000 ; 70$ : 13-6.

120. GUIL JL, TORIJA ME, GIMENEZ JJ, RODRIGUEZ I. Identification of fatty acids in edible wild plants by gas chromatography. / Chromatogr A 1996; 719 : 229-35.

121. LIU L, HOWE P, ZHOU YF, XU ZQ, HOWART C, ZHANG R. Fatty acids and beta-carotene in Australian purslane (Portulaca oleracea) varieties. I Chromatogr $A 2000 ; 893: 207-13$.

122. AYERZA R. Oil content and fatty acid composition of chia (Salvia hispanica L.) from five northwestern locations in Argentina. J Am Oil Chem Soc 1995; 72(9) : 1079-81.

123. COATES W, AYERZA R. Commercial production of chia in northwestern Argentina. J Am Oil Chem Soc 1998 ; 75(10) : 1417-20.

124. KALONJI E, DUMAS C, BERTA JL. Acides gras de la famille oméga 3 et système cardiovasculaire : intérêt nutritionnel et allégations. France : Agence Française de Sécurité Sanitaire des Aliments, 2003.

125. VERMOREL M, PITZ P, TAPPY L, LAVILLE M. Energie. In : Martin A, ed. Apports nutritionnels conseillés pour la population française. Paris : Lavoisier Tec \& Doc, 2001 : 17-36.

126. VOLATIER JL. Enquête INCA (Individuelle et Nationale sur les Consommations Alimentaires). Paris : Editions Tec \& Doc, 2000.

127. National cholesterol education program expert panel on detection, evaluation and treatment of high blood cholesterol in adults (Adult Treatment Panel III). Third report of the National Cholesterol Education Program (NCEP) expert panel on detection, evaluation and treatment of high blood cholesterol in adults (adult treatment panel III) : Final report. Circulation $2002 ; 106: 3143-421$.

128. KRAUSS RM, ECKEL RH, HOWARD B, ET AL. AHA dietary guidelines. Revision 2000 : A statement for healthcare professionals from the nutrition committee of the American Heart Association. Stroke 2000 ; 31 : 2751-66.

129. Food Gouv UK. <http ://www.food.gov.uk/science/research/researchinfo/ nutritionresearch/dietandcardiovasc/n02programme/n02projilist/n02015/ n02016/> date de consultation : 22/08/07.

130. Conseil supérieur d'hygiène publique de France. NOR : MESP9830614V Avis du 7 juillet 1998 du Conseil supérieur d'hygiène publique de France relatif à l'emploi d'huile de périlla en alimentation humaine (section de I'alimentation et de la nutrition). France : Direction Générale de la Santé. 1998. 
131. HIRSCH M. Saisine $n^{\circ}$ 2003-SA-0100 - Avis de l'Agence française de sécurité sanitaire des aliments relatif à l'évaluation de l'emploi de l'huile de lin, nature ou en mélange, dans l'alimentation courante ainsi que son intérêt nutritionnel en matière d'apport d'acide $\alpha$-linolénique. France : Agence Française de Sécurité Sanitaire des Aliments, 2003.

132. HIRSCH M. Saisine $n^{\circ}$ 2003-SA-0396 - Avis de l'Agence française de sécurité sanitaire des aliments relatif à l'évaluation de l'emploi de l'huile de lin, nature ou en mélange, dans l'alimentation courante ainsi que son intérêt nutritionnel en matière d'apport d'acide $\alpha$-linolénique. France : Agence Française de la Sécurité Sanitaire des Aliments, 2003.
133. HIRSCH M. Saisine $n^{\circ}$ 2004-SA-0409 - Avis de l'Agence française de sécurité sanitaire des aliments relatif à l'évaluation de l'emploi de l'huile de lin, nature ou en mélange, dans I'alimentation courante ainsi que son intérêt nutritionnel en matière d'apport d'acide $\alpha$-linolénique. France : Agence Française de Sécurité Sanitaire des Aliments, 2006.

134. TCHANKOU LEUDEU BT. Etude des propriétés nutritionnelles des huiles des oléagineux non-conventionnels : cas schweinfurthii Engl., Dacryodes edulis (G. Don) H. J. Lam, Ricinodendron heudelotii (Bail.) Pierre ex Pax, Tetracarpidium conophorum Müll. Arg. Hutch. and Dalz. Thèse, Université de Ngaoundéré, Cameroun. 2006. 